\title{
The hydrodynamics of water-walking arthropods
}

\author{
DAVID L. HU† AND JOHN W. M. BUSH \\ Department of Mathematics, Massachusetts Institute of Technology, Cambridge, MA 02139, USA
}

(Received 24 May 2008; revised 9 September 2009; accepted 11 September 2009)

We present the results of a combined experimental and theoretical investigation of the dynamics of water-walking insects and spiders. Using high-speed videography, we describe their numerous gaits, some analogous to those of their terrestrial counterparts, others specialized for life at the interface. The critical role of the rough surface of these water walkers in both floatation and propulsion is demonstrated. Their waxy, hairy surface ensures that their legs remain in a water-repellent state, that the bulk of their leg is not wetted, but rather contact with the water arises exclusively through individual hairs. Maintaining this water-repellent state requires that the speed of their driving legs does not exceed a critical wetting speed. Flow visualization reveals that the wakes of most water walkers are characterized by a series of coherent subsurface vortices shed by the driving stroke. A theoretical framework is developed in order to describe the propulsion in terms of the transfer of forces and momentum between the creature and its environment. The application of the conservation of momentum to biolocomotion at the interface confirms that the propulsion of water walkers may be rationalized in terms of the subsurface flows generated by their driving stroke. The two principal modes of propulsion available to small water walkers are elucidated. At driving leg speeds in excess of the capillary wave speed, macroscopic curvature forces are generated by deforming the meniscus, and the surface behaves effectively as a trampoline. For slower speeds, the driving legs need not substantially deform the surface but may instead simply brush it: the resulting contact or viscous forces acting on the leg hairs crossing the interface serve to propel the creature forward.

\section{Introduction}

We present here the results of an extensive series of experiments aimed at elucidating the propulsion mechanisms of water-walking arthropods. Our study is motivated principally by fundamental interest, specifically a desire to rationalize a number of nature's designs. Nevertheless, owing to the scales involved, this class of problems may serve to inspire and inform the biomimetic design of microfluidic devices. For example, the dynamic interaction between water-repellent solids and fluids is a problem of considerable interest in a number of engineering applications, for example the design of self-cleaning and drag-reducing surfaces (Bush, Hu \& Prakash 2008; Quéré 2008).

There has been considerable work reported in the biology literature on waterwalking insects, beginning with that of Aldrovandi (1618) and Ray (1710). The

$\dagger$ Present address: Departments of Mechanical Engineering and Biology, Georgia Institute of Technology, Atlanta, GA 30318, USA

$\ddagger$ Email address for correspondence: bush@math.mit.edu 
introduction of the concept of surface tension (Plateau 1873) provided the physical basis for understanding the weight support of small water-walking insects (Brocher 1910; Baudoin 1955), and the static equilibria of water-walking insects is now well understood (Mansfield, Sepangi \& Eastwood 1997; Keller 1998). The dynamics of water-walking arthropods is subtler and requires consideration of both the surface chemistry and the texture responsible for the creature's water repellency (Bush et al. 2008) and the interfacial fluid dynamics generated by its leg stroke (Bush \& Hu 2006).

The form and function of the surface layer (or 'integument') of water-walking creatures has been reviewed in Bush et al. (2008), and the dynamical significance of the hair geometry has been demonstrated in Prakash \& Bush (submitted). Waterwalking arthropods are water repellent by virtue of their waxy surface coating that increases the contact angle beyond $\pi / 2$ (Holdgate 1955), and a surface roughness that consists of a dense array of hairs (Andersen 1977; Gao \& Jiang 2004; Stratton, Suter \& Miller 2004b). When adjoining the free surface, these hairs trap air pockets, preclude bulk wetting of the leg and so maintain the leg's water repellency. When the leg is moving, however, the manner in which water repellency is maintained and force is transferred from the fluid to the driving legs is not immediately clear. We here demonstrate the means by which propulsive forces are generated by the driving stroke of water-repellent water-walking arthropods.

It has long been known that surface tension plays a critical propulsive role for most water-walking creatures (Dufour 1833; Brocher 1910; Baudoin 1955). With the invention of the high-speed camera in 1942, great strides were made towards describing their motion on the water surface. It became clear that water walkers possess a variety of gaits (Andersen 1976; Suter et al. 1997) that include walking, rowing and galloping. Locomotion by water walkers such as Microvelia, Mesovelia and Hydrometra is characterized by an alternating tripod gait analogous to that employed on land by cockroaches and other terrestrial hexapods (Altendorfer et al. 2001). The most efficient water walkers, such as water striders and fisher spiders, row using their middle pair of legs (Andersen 1976; Suter, Stratton \& Miller 2003; Stratton, Suter \& Miller 2004a). Bowdan (1978) noted that water striders revert to a walking gait on a fluid of higher viscosity. Fisher spiders switch from rowing to galloping in order to achieve their peak speed (Suter \& Wildman 1999). Certain water walkers can climb static menisci simply by deforming the free surface quasi-statically, thereby generating lateral capillary forces (Baudoin 1955; Miyamoto 1955; Hu \& Bush 2005). Finally, Microvelia and certain shore-dwelling creatures possess an emergency form of propulsion: by excreting surfactant, they generate surface-tension gradients that propel them short distances along the water surface (Linsenmair \& Jander 1976; Schildknecht 1976).

Darnhofer-Demar (1969) showed experimentally that water striders generate millimetre-scale indentations by striking the free surface and surmised that it was the associated curvature forces that provide the strider's thrust. He emphasized the presence of waves in the wake, and this fuelled work supporting the idea that wave drag on the driving leg plays a critical propulsive role (Andersen 1976; Denny 1993; Sun \& Keller 2001). This inference concerning the critical role of wave drag led to Denny's paradox (Suter et al. 1997), the proposal that infant water striders unable to generate waves should be incapable of self-propulsion (Denny 1993, 2004). Suter et al. (1997) performed a series of experiments in which the steady-state forces were measured on a spider leg suspended in a rotating flume. The authors estimated the relative magnitudes of forces on the leg owing to wave drag, fluid inertia and curvature forces; by demonstrating the persistence of the inertial forces even in the 
absence of waves, the authors effectively resolved Denny's paradox. Hu, Chan \& Bush (2003) argued that the propulsion of these creatures is most easily understood and the paradox most easily resolved by considering momentum transfer in their wake, an argument to be supported by the theoretical developments of $\S 5.2$. Hu et al. (2003) showed experimentally that the most common water-walking insect, the water strider, transfers momentum principally through subsurface vortices. In our experimental study reported in $\S 4$, we demonstrate that the generation of vortices by water walkers has a broad generality; moreover, we demonstrate that even in the absence of the generation of pronounced waves or vortices, water-walking insects are able to propel themselves by virtue of the microscale interaction between their rough integument and the water surface.

In $\S 5$, we develop a theoretical framework that may be used to integrate and rationalize our experimental observations of water-walking arthropods. Biolocomotion is generally rationalized in terms of the transfer of force and momentum between the creature and the surrounding fluid (Childress 1981). Conservation of momentum allows one to rationalize the high-Reynolds-number propulsion of fish (Wilga \& Lauder 2002) and birds (Spedding, Rosén \& Hedenstrom 2003) by considering the momentum transfer in their wakes, an approach of particular value when these wakes are characterized by coherent vortical structures. The motion of water walkers is complicated by the presence of the free surface. Implicit in the analysis of $\mathrm{Hu}$ et al. (2003) and Bühler (2007) for the water strider and in that of Hsieh (2004) for the basilisk lizard is that momentum is similarly conserved for propulsion at a free surface, a result to be proven in $\S 5.2$.

In this paper, we report micro- and macroscale observations of the different locomotory styles of water-walking arthropods. In $\S 2$, we describe our experimental techniques. In $\S 3$, we consider the state of wetting of the water walker's leg in both static and dynamic states. In $\S 4$, we characterize the leg stroke and the resulting dynamics of the underlying water. We proceed in $\S 5$ by considering the hydrodynamics of water-walking arthropods from a theoretical perspective, characterizing the hydrodynamic force and momentum transfer between the insect, the fluid and the free surface. Lastly, in $\S 6$ we discuss the implications of our work and suggest directions for future research.

\section{Experimental techniques}

Six water-walking and 20 terrestrial insects and spiders, common to our area, exhibited a range of water-walking techniques (figure 1). Freshwater walkers were gathered from Fresh Pond, Massachusetts; marine water walkers from the coast at Rockport, Massachusetts; and terrestrial insects from Cambridge, Massachusetts. These creatures were raised in captivity in aquaria, sustained on a diet of grounddwelling insects.

Insect leg widths were measured using several light microscopes (SKope 3000100 by Boreal, LSM Pascal confocal microscope by Zeiss and stereo microscope STEMI 2000 by Zeiss); their microscale hair coverings were examined with a scanning electron microscope (XL30 ESEM by FEI). We filmed the insects using a digital still camera (Sony DSC-F707), a digital video camera (Sony DCR-TRV950) and a high-speed digital video camera (Redlake Motionscope PCI 8000). High-speed films were digitized using Midas motion analysis software. Insects were filmed at 1/1000 s exposure time at 30-500 f.p.s. Plan views of their locomotion were filmed in a shallow 

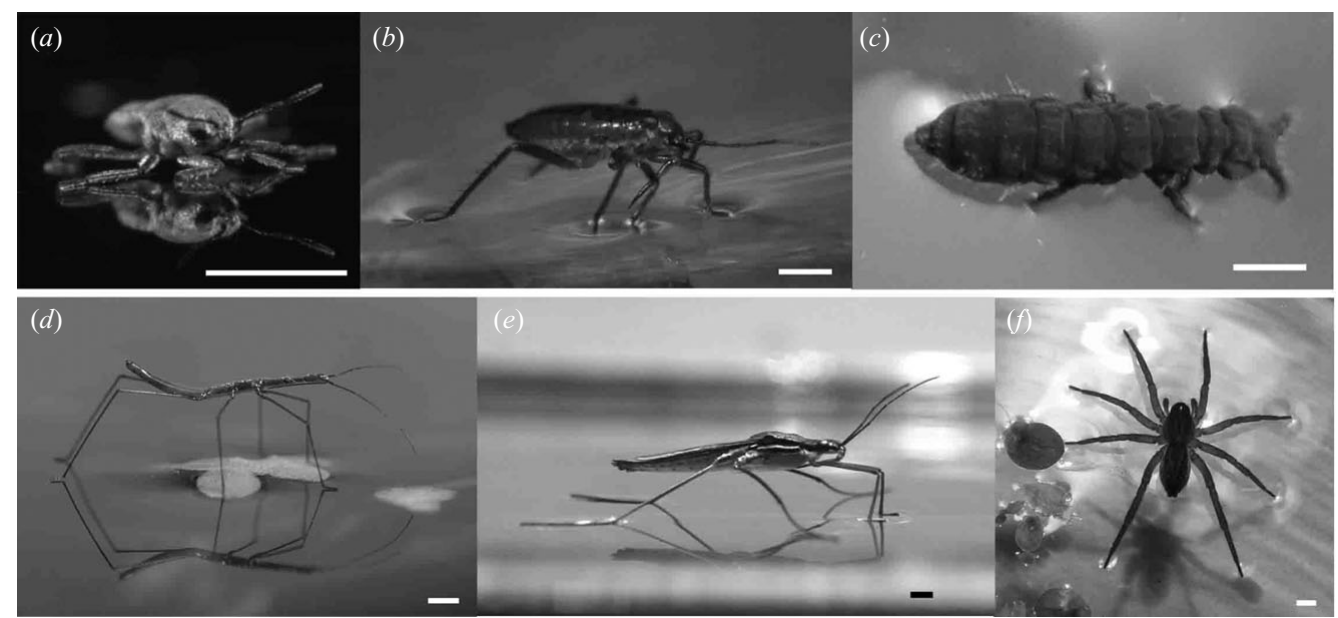

FIGURE 1. The water-walking arthropods examined in our experimental study, ordered by size: $(a)$ the broad-shouldered water strider Microvelia, $(b)$ the water treader Mesovelia, $(c)$ the springtail Anurida maritima, $(d)$ the water measurer Hydrometra stagnorum, $(e)$ the water strider Gerris, $(f)$ the fisher spider Dolomedes triton. Scale bars, $1 \mathrm{~mm}$.

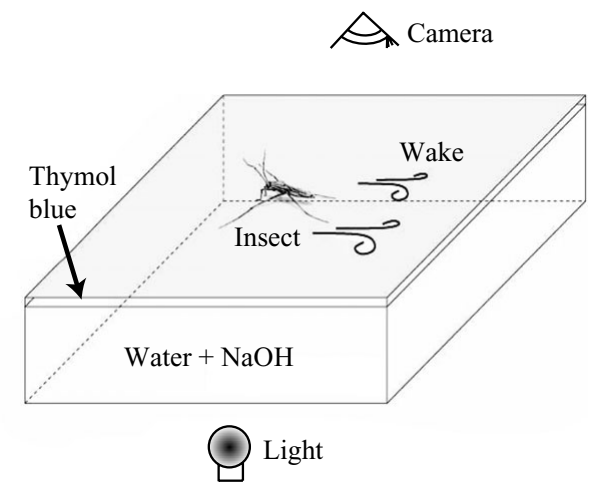

FIGURE 2. The experimental apparatus used for flow visualization. A mildly basic solution is illuminated from below and viewed from above. The motion of an opaque tracer, Thymol Blue dye, is used to track the motion of the flows generated by water-walking insects.

tank $(15 \mathrm{~cm} \times 15 \mathrm{~cm} \times 4 \mathrm{~cm})$ and side views in a slender tank $(3 \mathrm{~cm} \times 20 \mathrm{~cm} \times 20 \mathrm{~cm})$ that constrained the path of the insect so that it remained in focus.

Flow visualization was accomplished in a series of particle tracking and dye studies using the apparatus illustrated in figure 2. Particle tracking was performed by lighting the subject from above, placing a dark background beneath the tank and seeding the fluid with either Kalliroscope AQ-1000 or Pliolite particles (S-6B, Goodyear Chemicals), ground to a grain size of 50-100 $\mu \mathrm{m}$. A Thymol Blue technique (Voropayev \& Afanasyev 1994) was used to visualize subsurface flows. The shallow tank was lit from below using a light table (LightTracer, Artograph) and filled to a depth of $0.5-1 \mathrm{~cm}$ with a weak dilution of sodium hydroxide $(\mathrm{pH} 9.2)$. Thymol Blue powder was sprinkled on the surface in the path of the insect; the insect propelled itself through the dye field as the dye sank to the bottom of the tank. The Thymol Blue prompted Marangoni convection rolls of width comparable to the depth of the dye layer (Scriven \& Sternling 1970). The graininess of these convection rolls could 

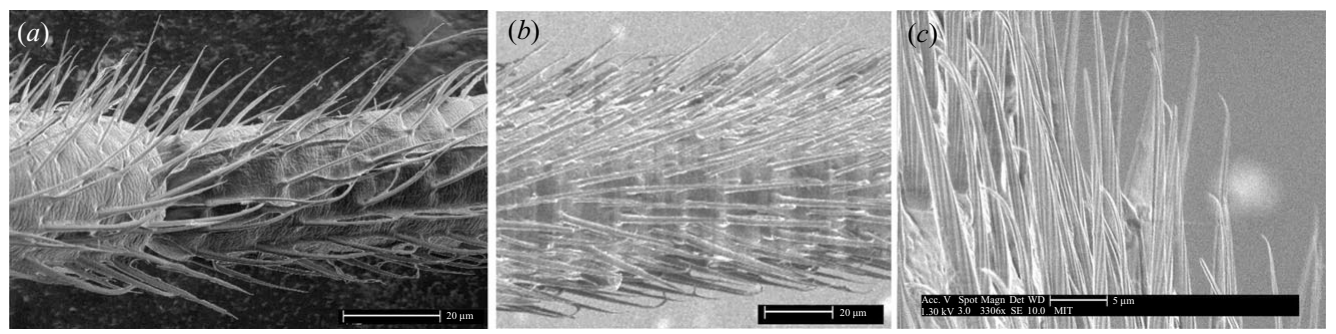

FIGURE 3. Scanning electron microscope images of the hair layer on the driving legs of Mesovelia ( $a$, scale bar $20 \mu \mathrm{m})$ and the water strider ( $b$, scale bar $20 \mu \mathrm{m} ; c$, scale bar $5 \mu \mathrm{m}$ ). The leg is a hairy brush whose hairs are tilted at $30^{\circ}$ to the leg surface. Hairs are typically $30 \mu \mathrm{m}$ long, $1-3 \mu \mathrm{m}$ thick at the base and tapered; their density is $12000-16000$ hairs $\mathrm{mm}^{-2}$. (c) A closer view of the hairs shows that their tips are bent inward towards the legs; moreover, each hair is patterned with grooves of characteristic width $400 \mathrm{~nm}$ that run its length. Images courtesy of Manu Prakash.

be reduced by stirring the fluid. Stirring also allowed the dye-based mixture to be used repeatedly.

We also observed the deflection of the free surface by the insect leg. The leg was never observed to penetrate the free surface. Instead, a water-repellent state was maintained: a thin air layer remained trapped in its integument (Bush et al. 2008). Some insects are also able to pull up on the free surface by virtue of their hydrophilic claws (Baudoin 1955; Noble-Nesbitt 1963; Andersen 1976), an important adaptation for meniscus climbing ( $\mathrm{Hu} \&$ Bush 2005). The sign of the surface deflection was determined using a lighting technique adopted from Baudoin (1955). By lighting directly from above the insect, the surface deflection could be inferred qualitatively from the manner in which light was focused onto the tank bottom. Depressions of the free surface defracted light radially outward and were marked by dark spots on the tank bottom; conversely, peaks in the free surface were marked by bright spots (figures 11, 14 and 16).

The insects were encouraged to move using a variety of techniques. When the room was dark, a beam of light produced by a flashlight would attract the insects. Prodding with a wire, shaking the tank or blowing on the insect were also effective in encouraging it to move in a preferred direction or, in the case of Microvelia, to secrete surfactant.

\section{Microscale considerations}

The macroscopic flows generated by the driving stroke of water-walking insects will be considered in $\S \S 4$ and 5. We first consider the microscopic interaction between the arthropod cuticle and the interface, specifically its role in water repellency $(\S 3.1)$ and propulsion $(\S 3.2)$.

\subsection{Water repellency}

Water-walking arthropods are covered with a dense hair mat that renders them water-repellent (Figure 3; Bush et al. 2008). The bodies of water walkers have two distinct hair layers, namely the macrotrichia for waterproofing the insect on the water surface and the shorter microtrichia for trapping air should the insect be submerged by raindrops or a crashing wave (Thorpe \& Crisp 1947; Hinton 1976). We note that in the case of submergence, the air trapped in the microtrichia serves both as a buoy and an external gill that for certain arthropods enables underwater breathing 


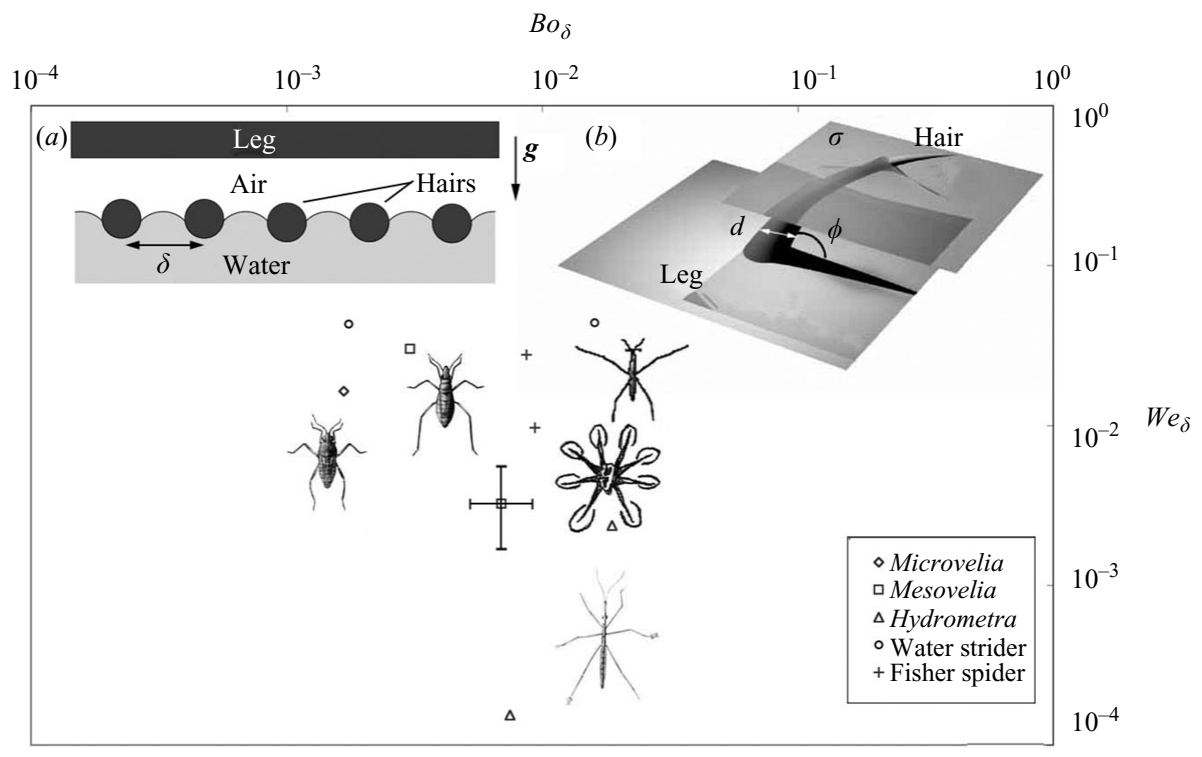

FIGURE 4. The roughness Weber number $W e_{\delta}=\rho U^{2} \delta / \sigma$ and Bond number $B o_{\delta}=\rho g \delta^{2} / \sigma$ characterizing the water repellency of the hair layers of five species of water-walking insects and spiders. Here $U$ represents the peak leg speed and $\delta$ the inter-hair spacing. All water walkers are characterized by low Weber number, indicating that their driving legs remain in a Cassie state as they propel themselves on the water surface. Moreover, the low Bond number indicates that the air layers between the hairs remain intact in the face of hydrostatic pressures generated by the driving stroke. (a) A cross-sectional view of an array of hairs with spacing $\delta$ lying tangent to the free surface. (b) Shows an oblique view of a single hair of length $L$, width $d$ and angle $\phi$ piercing the free surface.

(Flynn \& Bush, 2008). We proceed by investigating the characteristics of the hairs on the driving legs.

The surface of the water-walking arthropod leg consists of a mat of macrotrichia tilted towards the leg tips; the tilt angle $\phi$ and spacing $\delta$ vary among species (figure $4 a$ ). The leg hairs of Microvelia are shown in figure 3(a) and schematically in figure 5. The hairs generally have a length $L$ of $20-60 \mu \mathrm{m}$ and a diameter $d$ of 1-2 $\mu \mathrm{m}$ at the base and taper to a point at their tip. Inter-species variation in the hair tilt and spacing are shown in table 1. Figure 6(a) shows that the leg of Mesovelia resembles a hairy brush. A view from below (figure 7) shows that the arthropod leg traps air within its integument, thus maintaining a water-repellent state. These air pockets can be seen as a silvery envelope around the legs when they strike the water surface as is the case for the fisher spider (figure 8). We proceed by rationalizing the maintenance of this air layer in both static and dynamic settings.

The waxy material covering the integument of water-walking arthropods has a chemical contact angle of $\theta_{e}=105^{\circ}$ (Holdgate 1955) and so is hydrophobic. Because $\theta_{e}>\pi / 2$, the addition of surface roughness increases the energetic cost of wetting and so discourages wetting (Dussan 1979; de Gennes, Brochard-Wyart \& Quére 2003). The form of contact between a rough, hydrophobic solid and water depends explicitly on the form of the roughness and the fluid pressure. With moderate roughness, the water entirely wets the substrate, yielding a Wenzel state (Figure $9 c$; Wenzel 1936). When the roughness is increased sufficiently, it is energetically favourable for air inclusions to be trapped within the rough surface, so that the solid is wetted only at 


\begin{tabular}{llrrccc}
\hline \multirow{2}{*}{ Gait } & Species & $\begin{array}{c}n\left(\text { hairs cm }^{-2}\right) \\
\text { Hair density }\end{array}$ & $\begin{array}{c}L(\mu \mathrm{m}) \\
\text { Length }\end{array}$ & $\begin{array}{c}d(\mu \mathrm{m}) \\
\text { Width }\end{array}$ & $\begin{array}{c}\phi(\mathrm{deg} .) \\
\text { Tilt angle }\end{array}$ & $\begin{array}{c}\delta(\mu \mathrm{m}) \\
\text { Spacing }\end{array}$ \\
Rowing & Strider & $1.2-1.6 \times 10^{6}$ & $20-40$ & $1.5-2$ & $30-50$ & 7 \\
& Velia & $1 \times 10^{6}$ & $30-40$ & $1-2$ & $50-60$ & - \\
& Halobates & $0.8-1.2 \times 10^{6}$ & $20-30$ & 1 & $20-40$ & - \\
\multirow{5}{*}{ Walking } & Fisher spider & $2.5-3.6 \times 10^{5}$ & - & 6 & - & 13 \\
& Mesovelia & $4 \times 10^{5}$ & $30-60$ & $2-3$ & 50 & 10 \\
& Hydrometra & $2-3 \times 10^{5}$ & 15 & 5 & 90 & 15
\end{tabular}

TABLE 1. Tarsal hair properties of water-walking insects and spiders. Leg hairs of length $L$, width $d$ and angle $\phi$ with respect to the leg surface are arranged with density $n$ and spacing $\delta$. Data reprinted from Andersen $(1976,1977)$; spider data from Stratton et al. (2004b).
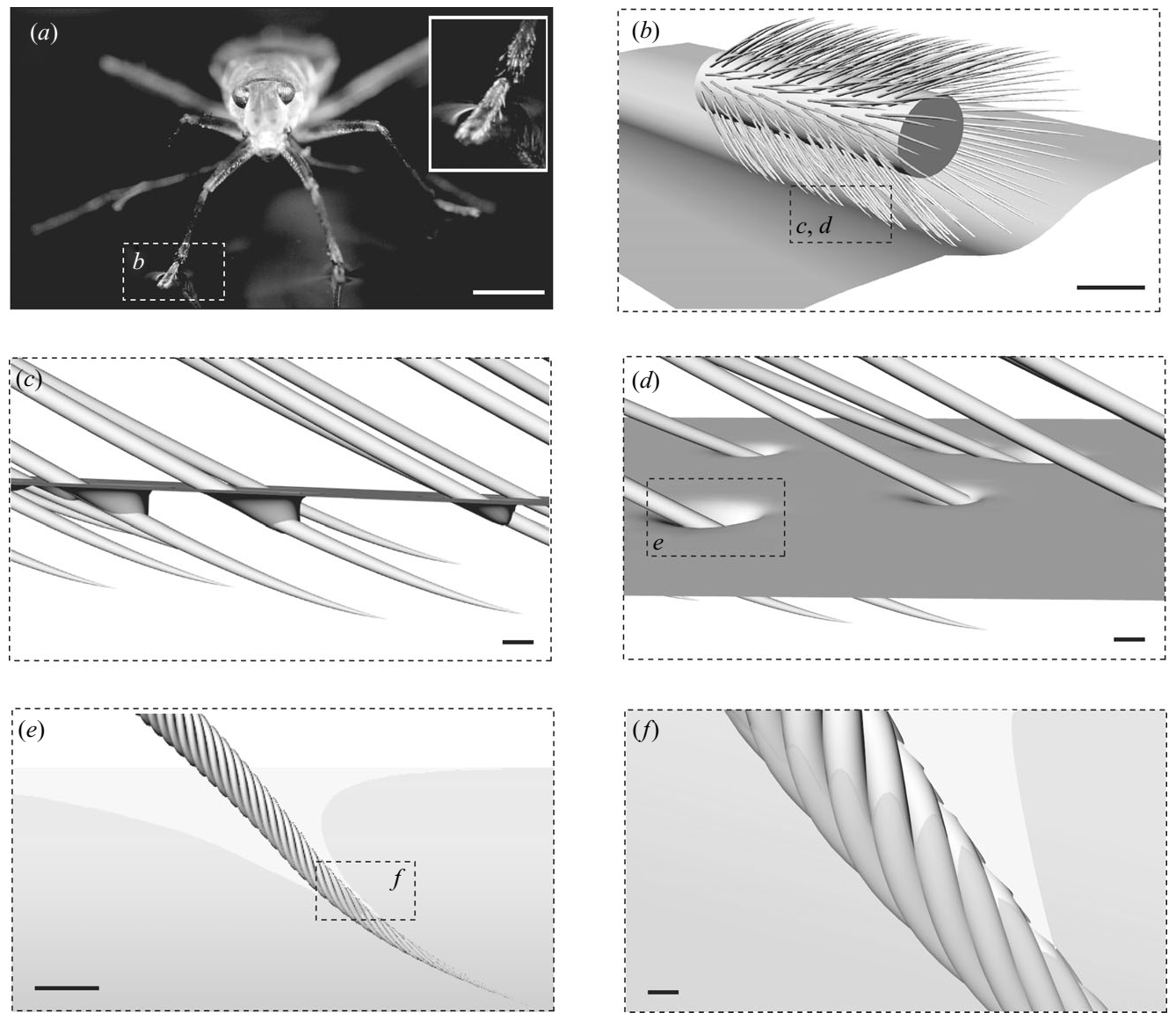

Figure 5. The contact between the water treader Mesovelia and the free surface. (a) Mesovelia supports its weight by deforming the surface. Scale bar, $1 \mathrm{~mm}$. (b) A schematic of a section of a hairy leg. Scale bar, $100 \mu \mathrm{m}$. $(c, d)$ Further schematics of individual hairs penetrating the surface. Scale bar, $1 \mu \mathrm{m}$. (e) A single hair penetrating the free surface. Scale bar, $1 \mu \mathrm{m} .(f)$ The hairs are covered in nanogrooves that trap air when the hair is submerged. Scale bar, $0.1 \mu \mathrm{m}$.

the extremities of its roughness elements. The apparent contact angle $\theta^{*}$ of a drop in the resulting water-repellent or 'Cassie' state is given by the Cassie-Baxter relation (Cassie \& Baxter 1944)

$$
\cos \theta^{*}=f_{s}-1+f_{s} \cos \theta
$$



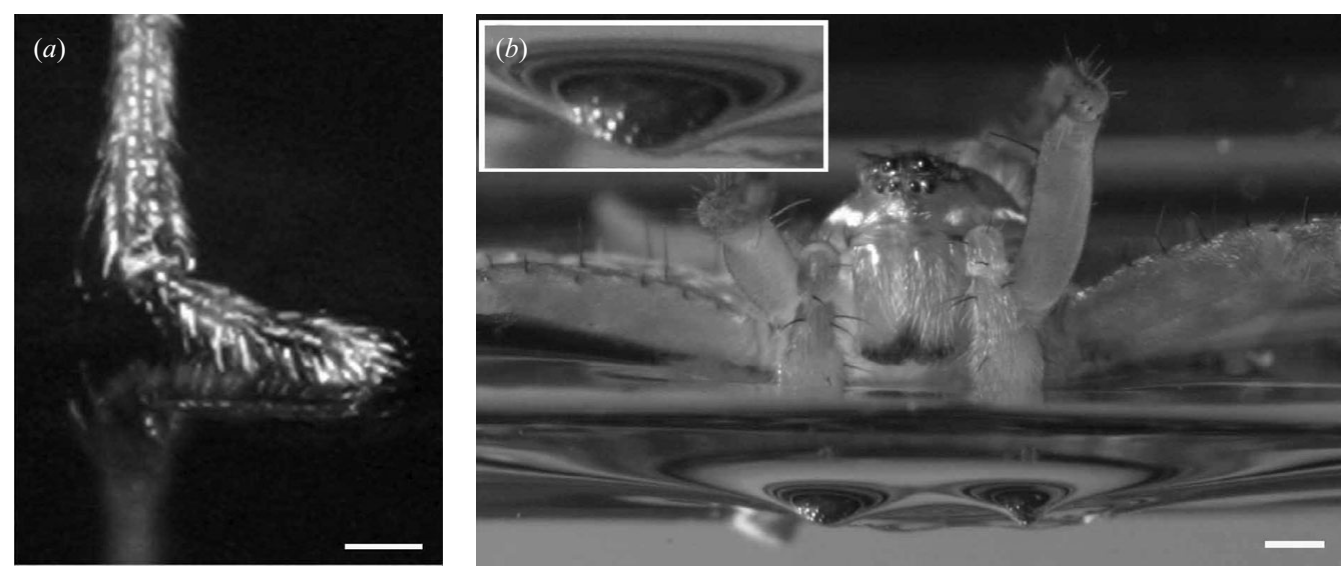

FIgure 6. The hairy legs of $(a)$ Mesovelia and $(b)$ the fisher spider. As is evident in the inset of $(b)$, the hairs maintain an array of air pockets when pressed against the water surface. Scale bars: (a) $100 \mu \mathrm{m},(b) 0.15 \mathrm{~cm}$.

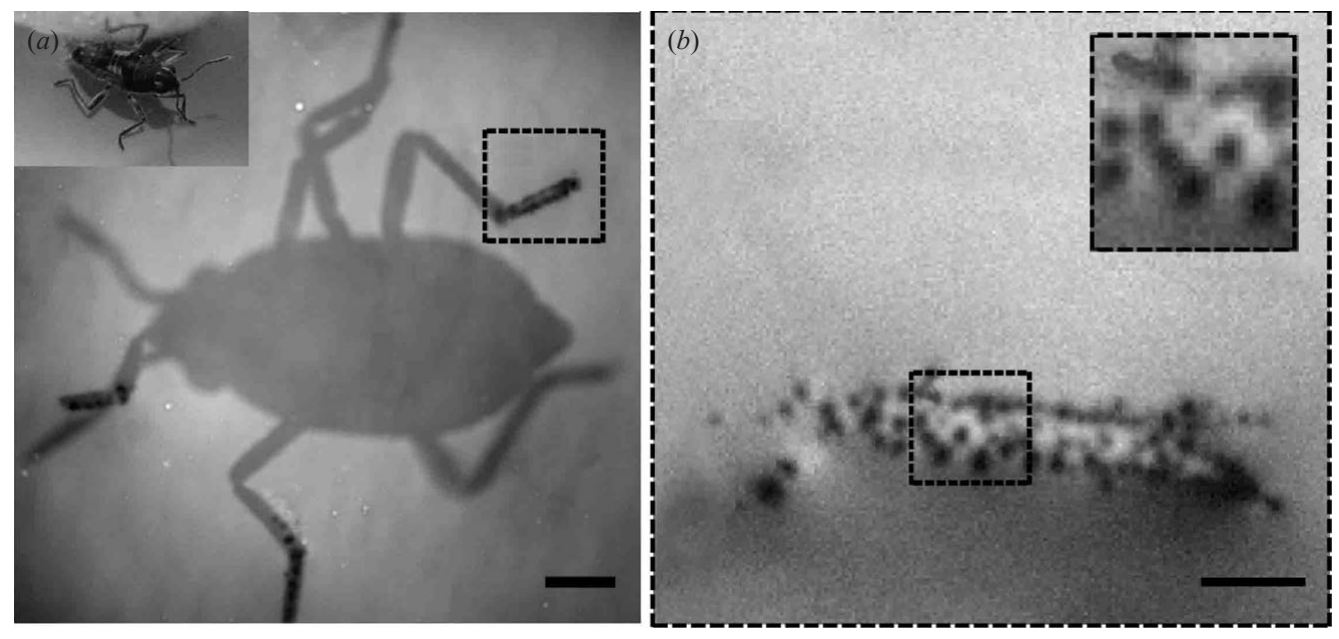

FiguRE 7. Brightfield images of water-walking arthropods in a Cassie state, as seen from below through an inverted microscope. (a) A live Microvelia standing on the water surface grooming its water-repellent legs. Scale bar $400 \mu \mathrm{m}$. A closer look at the supporting foot (the hatched box in $a$, magnified in $b$ and its inset) shows individual hair pinholes corresponding to the contact lines between the cuticle and the water surface. Scale bars, $100 \mu \mathrm{m}$. Images courtesy of Manu Prakash.

where $f_{s}$ is the exposed area fraction of the solid substrate (e.g. the ratio of the area of the pillar tops to total base area in figure $9 d$ ). For sufficiently small $f_{s}$, the effect of the texture is to increase the contact angle dramatically from $\theta$ to $\theta^{*}$ and so qualify the integument as being superhydrophobic $\left(\theta^{*}>150^{\circ}\right)$. Gao \& Jiang (2004) measured the contact angles of water strider integument to be $168^{\circ}$ and rationalized this high value by virtue of the nanogrooves on the hairs. Stratton et al. (2004b) reported values of $152^{\circ}$ for fisher spiders. Contact angles for a variety of water-walking arthropods are reported in Bush et al. (2008).

We note that a consequence of the hydrophobicity via texturing of the integument of water-walking arthropods is that if the leg is immersed in a fluid with which it 

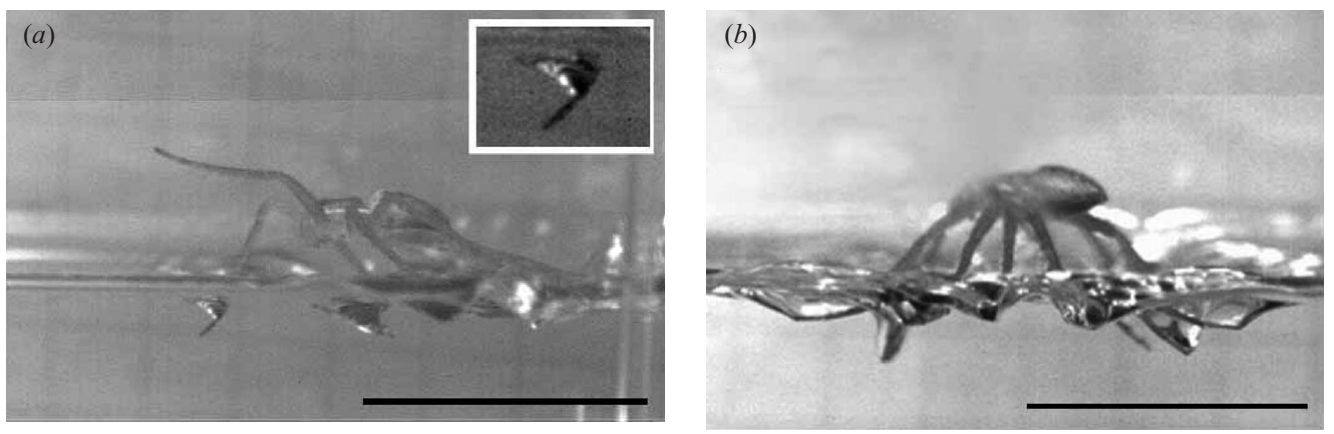

FiguRE 8. High-speed images of the fisher spider $(a)$ galloping and $(b)$ leaping by driving its legs against the free surface. In $(a)$, its leg penetrates the free surface, as shown in the close-up view in the inset, but maintains a Cassie state. Scale bars, $7 \mathrm{~mm}$.
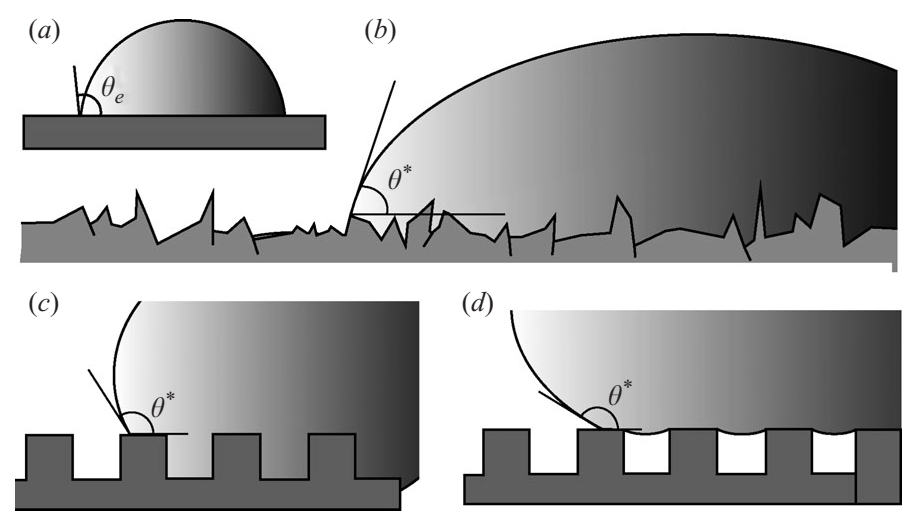

FIGURE 9. Roughening a surface will amplify its wetting tendencies: $(a) \theta_{e}$ defines the equilibrium or chemical contact angle on a flat surace. $(b)$ On a rough surface, the microscopic contact angle remains $\theta_{e}$, but the observed contact angle on the macroscopic scale, $\theta^{*}$, depends explicitly on the surface roughness. The two generic states of wetting, the Wenzel and Cassie states, are shown schematically in $(c)$ and $(d)$. In the Wenzel state, the pores are impregnated by fluid, increasing the fluid-solid contact. In the Cassie state, air pockets are trapped by the overlying fluid, reducing the fluid-solid contact.

has a low contact angle $\left(\theta<90^{\circ}\right)$, such as soapy water, the hairy leg becomes superhydrophilic and so imbibes water. The sensitivity of water-walking arthropods to surfactant was simply observed: the addition of detergent to the water surface caused water striders to sink through the interface. Note that the sinking is not simply due to the loss of static weight support by lowering of surface tension, since water walkers have a high margin of safety (Hu et al. 2003; Bush et al. 2008). Instead, the sinking is caused by the surfactant eliminating the water repellency of the integument, altering it from a non-wetting Cassie state to a wetting Wenzel state.

The maintenance of a Cassie state on the hairs is a fundamental constraint on water-walking arthropods; otherwise, they sink through the interface. The criterion for wetting is that the ambient pressure should be less than the Laplace pressure generated by this intrusion of water into the cuticle. Specifically, the dynamic pressure $\rho U^{2}$ generated by a leg stroke at speed $U$ and the hydrostatic pressure $\rho g L$ associated with submergence to a depth $L$ must be less than $\Delta P=\sigma / \delta$, where $\delta$ is the characteristic lateral scale of the cuticle roughness (or $\Delta P=\sigma h / \delta^{2}$ if the roughness amplitude $h<\delta$ ) 
(a)
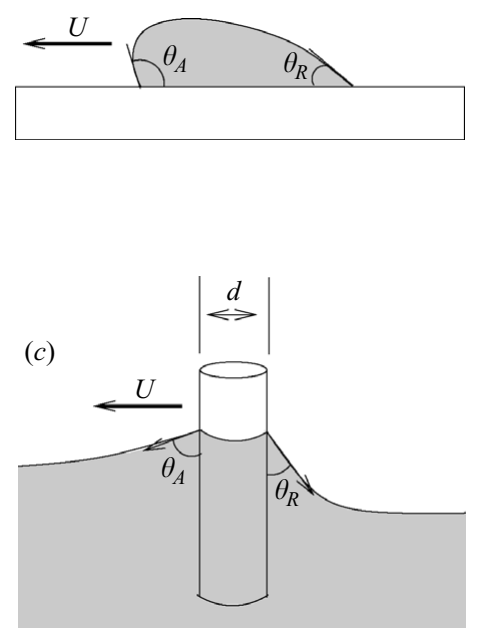

(b)
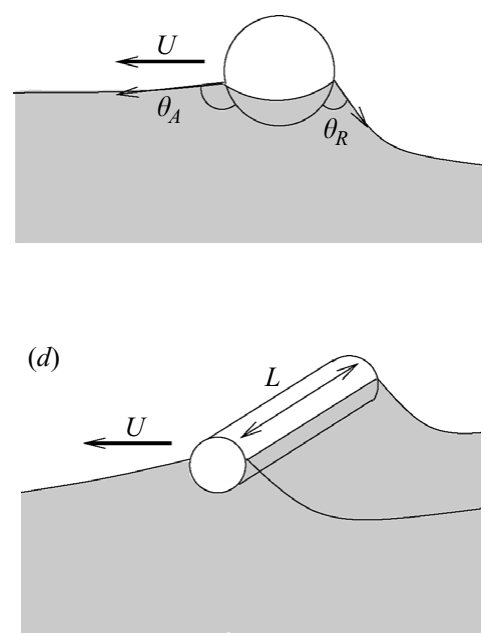

FIgURE 10. Contact angle hysteresis. (a) A drop sitting on a surface may exhibit a range of static contact angles between the receding and advancing values, respectively $\theta_{R}$ and $\theta_{A} \cdot(b)$ Similarly, if a small spherical body is dragged through a fluid, the maximum steady surface tension force resisting the motion will scale with $\sigma\left(\cos \theta_{A}-\cos \theta_{R}\right)$. Cylindrical bodies will also be subject to contact forces, but their magnitudes necessarily depend on their orientation with respect to the surface. $(c)$ If the cylinder is vertical, the horizontal component of the contact force scales as the contact perimeter, $w$. $(d)$ If the cylinder is horizontal, the contact force will depend on the direction of motion, scaling as $w$ in the axial direction and $L$ in the transverse.

(Bartolo et al. 2006; Reyssat et al. 2006). The relative magnitudes of these dynamic, hydrostatic and curvature pressures are prescribed by the roughness Weber and Bond numbers, namely $W e_{\delta}=\rho U^{2} \delta / \sigma$ and $B o_{\delta}=\rho g L \delta / \sigma$, where $L$ now represents a characteristic leg length. The values of $W e_{\delta}$ and $B o_{\delta}$ for the water walkers considered in our study are given in figure 4 . Note that none of these creatures is at risk of wetting under the influence of either dynamic or hydrostatic pressures. For a hair separation distance of $10 \mu \mathrm{m}$, the impregnation pressure for water striders is nearly $0.06 \mathrm{~atm}$, indicating that dive depths of $70 \mathrm{~cm}$ are possible before the air layer trapped by their integument collapses. We note that insects specialized for subsurface breathing can typically ascend to greater depths (Vogel 2006; Flynn \& Bush, 2008). The wetting speed for the water strider is given by $U_{w} \sim 300 \mathrm{~cm} \mathrm{~s}^{-1}$, approximately three times the peak speed of its driving leg. In terms of wetting, the greatest danger posed to water-walking arthropods is thus raindrops, whose impact speed may be as high as $1000 \mathrm{~cm} \mathrm{~s}^{-1}$ (Spilhaus 1948). We noted that when water striders did become wetted, they would seek out dry land, where their integument would dry through evaporation after a time of approximately $1 \mathrm{~min}$.

\subsection{Force transmission}

In $\S 5$, we shall characterize the hydrodynamic force generated by the leg of a water walker striking the free surface and so shall provide a macroscopic description of propulsion at the interface. Here, we consider the microscale interaction of the integument with the free surface. Specifically, we consider the relative magnitudes of the components of the hydrodynamic force on the individual hairs via scaling. An important contributor to the force generated by the leg stroke are the contact forces resulting from contact angle hysteresis (figure 10; de Gennes et al. 2003). When a fluid drop wets a solid, the static contact angle is bound above and below by, 
respectively, the advancing and retreating angles $\theta_{a}$ and $\theta_{r}$. As a drop of radius $R$ rolls along a solid substrate, its leading edge advances at $\theta_{a}$ and its trailing edge retreats at $\theta_{r}<\theta_{a}$; consequently, its motion is resisted by a contact force proportional to $2 \pi R\left(\cos \theta_{a}-\cos \theta_{r}\right)=2 \pi R \Delta \cos \theta$ (figure $10 a$; Dussan \& Chow 1983). Similarly, when a solid brushes across a free surface, differences in contact angles on its leading and trailing edges will generally result in its motion being resisted by a contact force whose magnitude depends on both the body geometry and the contact angle hysteresis (figure $10 b-d$ ).

Consider a cylindrical leg hair with diameter $d \approx 1 \mu \mathrm{m}$, wetted length $L \approx 10 \mu \mathrm{m}$ and speed $U \sim 30 \mathrm{~cm} \mathrm{~s}^{-1}$ either puncturing the interface (figure 10c) or brushing it (figure $10 d$ ). Characteristic magnitudes of the various hydrodynamic forces may be written as

$$
\left.\begin{array}{l}
F_{\text {contact }}^{\perp} \sim \sigma d \Delta \cos \theta, \quad F_{\text {contact }}^{\|} \sim \sigma L \Delta \cos \theta, \quad F_{\text {buoyancy }} \sim \rho g L d^{2}, \\
F_{\text {viscous }} \sim \frac{\mu U}{d} d L, \quad F_{\text {inertia }} \sim \rho U^{2} d L .
\end{array}\right\}
$$

The contact forces depend explicitly on the orientation of the hair relative to the interface: hairs intruding normal and lying tangent to the interface will experience respective forces $F_{\text {contact }}^{\perp}$ and $F_{\text {contact }}^{\|}$. Note that the contact forces in general depend on the magnitude of the contact angle hysteresis through $\Delta \cos \theta=\cos \theta_{A}-\cos \theta_{R}$; however, for the sake of simplicity, we consider this quantity to be $O(1)$ and proceed with our scaling. The relative magnitudes of the various components of the hydrodynamic force acting on individual hairs can be written in terms of standard dimensionless groups, whose characteristic magnitudes are assessed by choosing $U=10 \mathrm{~cm} \mathrm{~s}^{-1}, d=10^{-4} \mathrm{~cm}$ and $L=30 d$ :

$$
\left.\begin{array}{l}
R e_{\text {hair }}=\frac{U d}{\nu} \sim 0.1, \quad B o_{\text {hair }}=\frac{\rho g d L}{\sigma} \sim 10^{-5}, \\
W e_{\text {hair }}=\frac{\rho U^{2} d}{\sigma} \sim 10^{-4}, \quad C a^{\|}=\frac{\mu U}{\sigma} \sim 10^{-2}, \quad C a^{\perp}=\frac{\mu U L}{\sigma d} \sim 1 .
\end{array}\right\}
$$

At speeds typical of water-walking insects, forces associated with the fluid inertia and buoyancy are dominated by contact and viscous forces, the relative magnitudes of which depend on the hair orientation.

The leg hairs on most arthropods are tilted (table 1). The most specialized water walkers, such as the water strider, have hair tips that curve to lie tangent to the water surface (figure 3). This tilting contributes positively to the water repellency of the integument (Thorpe \& Crisp 1947; Bush et al. 2008); moreover, as is evident in (3.3), it renders contact forces dominant relative to viscous forces. For such highly specialized water walkers, the microscopic interaction of their cuticle and the interface generates propulsive forces in the form of contact forces acting on individual hairs. Less specialized water walkers with integument that pierces rather than lies tangent to the interface can still generate thrust through some combination of contact and viscous forces.

By measuring the dyne-scale forces acting on a drop being dragged along the surface of a water strider's leg, Prakash \& Bush (submitted) demonstrated that the contact forces are anisotropic because of the tilted hair geometry, with contact forces being largest for motion perpendicular to the leg. This anisotropy may be rationalized with the above scaling (3.3) and yields insight into the orientation of the leg during the driving stroke and gliding phase. In particular, the leg strike perpendicular to the direction of motion typical of rowers maximizes thrust via contact forces. Their 
study also made clear that the water strider's cuticle is unidirectional by virtue of the flexibility of the individual hairs: contact forces are least for motion in the direction of hair tilt. Therefore, aligning their legs with the direction of motion reduces contact forces during the gliding phase (Bush et al. 2008).

An estimate for the total viscous stress resulting from the leg hairs poking through the interface is simply given by the product of the viscous force acting on an individual hair and the hair density $n: \tau_{v i s c o u s}=n \mu U L$. The viscous force generated by a leg with contact area $A$ brushing the free surface is thus given by $F_{b r u s h}^{\mu}=n \mu U L_{w} A$, where $L_{w}$ denotes the length of wetted hair. The analogous stress generated by contact forces during a brushing stroke is $F_{b r u s h}^{\sigma}=n \sigma \Delta \cos \theta L_{w} A$ for hairs lying tangent to the interface. We note that these viscous and contact forces are available to all water-walking arthropods, independent of their ability to substantially deflect the free surface on a macroscopic scale. This discussion of force transmission on the microscale will inform our experimental observations, as well as our analysis of the macroscopic fluid dynamics presented in $\S 5$.

\section{Modes of locomotion}

The physical variables characterizing the driving leg geometry and locomotion of the water walkers examined are reported in table 2. The associated dimensionless groups are defined in table 2, and their characteristic values are listed. The peak driving leg speeds $U$ indicate that all water walkers move at a high Reynolds number. Moreover, with the exception of the thick-legged fisher spider, for which $W e \sim 1$, the Weber numbers are generally small, indicating that curvature forces represent the principle propulsive force for water-walking arthropods. We note that these dimensionless variables are all based on the macroscopic properties of the driving leg. In $\S 5$, our assessment of the dominance of curvature forces in the propulsion will be reconsidered in light of the microscopic picture considered in $\S 3.2$.

A similar walking gait is used by Mesovelia, Microvelia and Hydrometra for propulsion on both land and water. The insects support their weight on a tripod of legs, as can be seen by the associated menisci in figure 11. During the driving stroke, the meniscus under each leg enlarges as it is driven backward, indicating that the insect is pushing both downward and backward. Flow visualization shows that the walking gaits of Hydrometra and Mesovelia generate two rows of dipolar vortices (e.g. figure $12 c$ ). We note that terrestrial insects such as ants, which use the alternating tripod gait, are generally poor water walkers owing to their sparse hair covering, which allows them to be wetted by the water and therefore entangled at the interface.

An inhabitant of shoreside rocks, Anurida maritima also uses an alternating tripod walking gait. Like the freshwater walkers, this marine insect is hexapedal and water repellent. However, it walks on the ends of its relatively smooth unguis or claws that provide very little traction; they present a small surface area, two thirds of which is hydrophobic (Janssens 2005). Compared with water walkers that contact the interface through their rough integument, its propulsion is remarkably inefficient (table 2). Flow visualization shows that the leg motion generates a distinctly different form of flow than that of other water walkers: rather than each leg strike generating a vortex, its collective leg motions generate a single jet that ultimately wraps up into a vortex (figure 11d).

The water strider rows at the free surface, its driving stroke propelling it forward either along or above the water surface. During the driving stroke of approximate duration $0.01 \mathrm{~s}$, the middle legs are driven back in a sweeping motion along the 


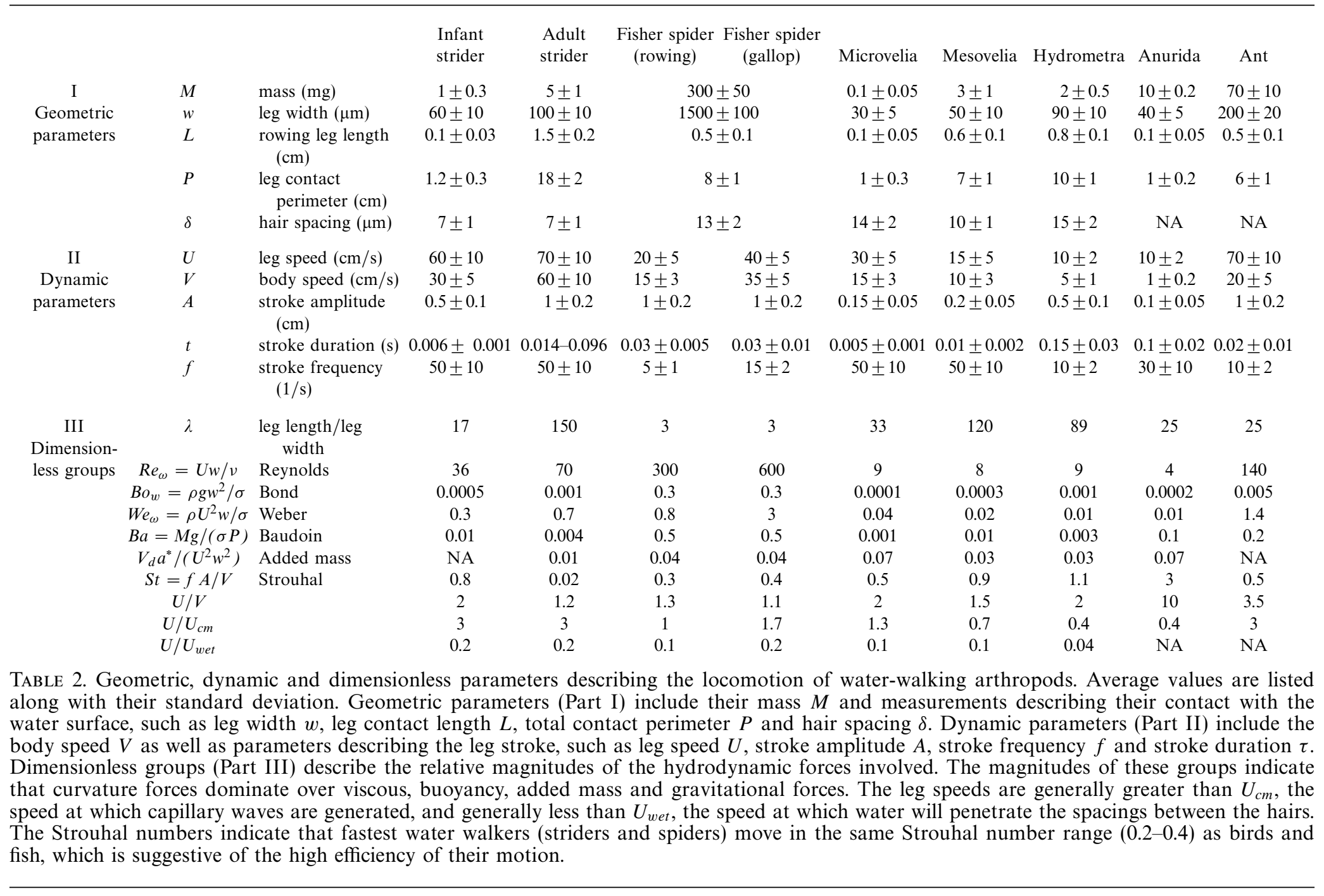



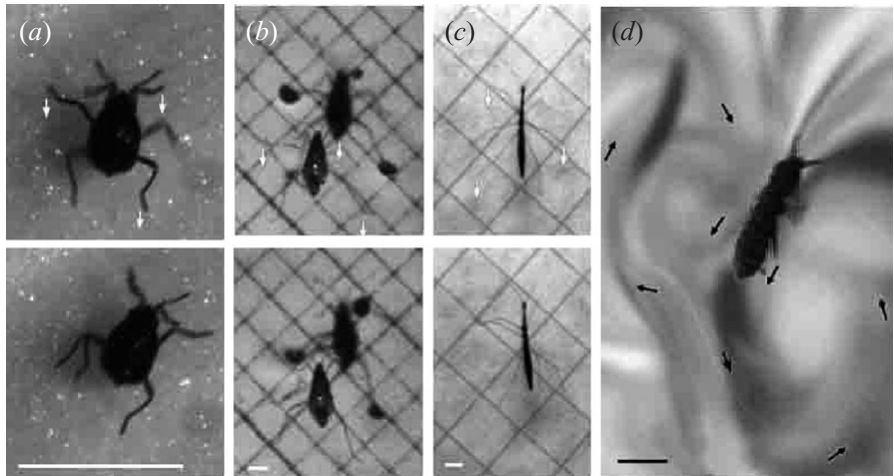

FIGURE 11. The alternating tripod gait of (a) Microvelia, $(b)$ Mesovelia, $(c)$ Hydrometra and $(d)$ Anurida. The insects support their weight on a tripod of legs and move forward by sweeping them backward. The time between the photos is $\Delta t=0.01 \mathrm{~s}$. In $(d)$, a single dipolar vortex is generated in the wake of Anurida, as visualized using Thymol Blue. White arrows indicate the direction of the leg motion and black arrows the direction of the flow. Scale bars, $2 \mathrm{~mm}$.
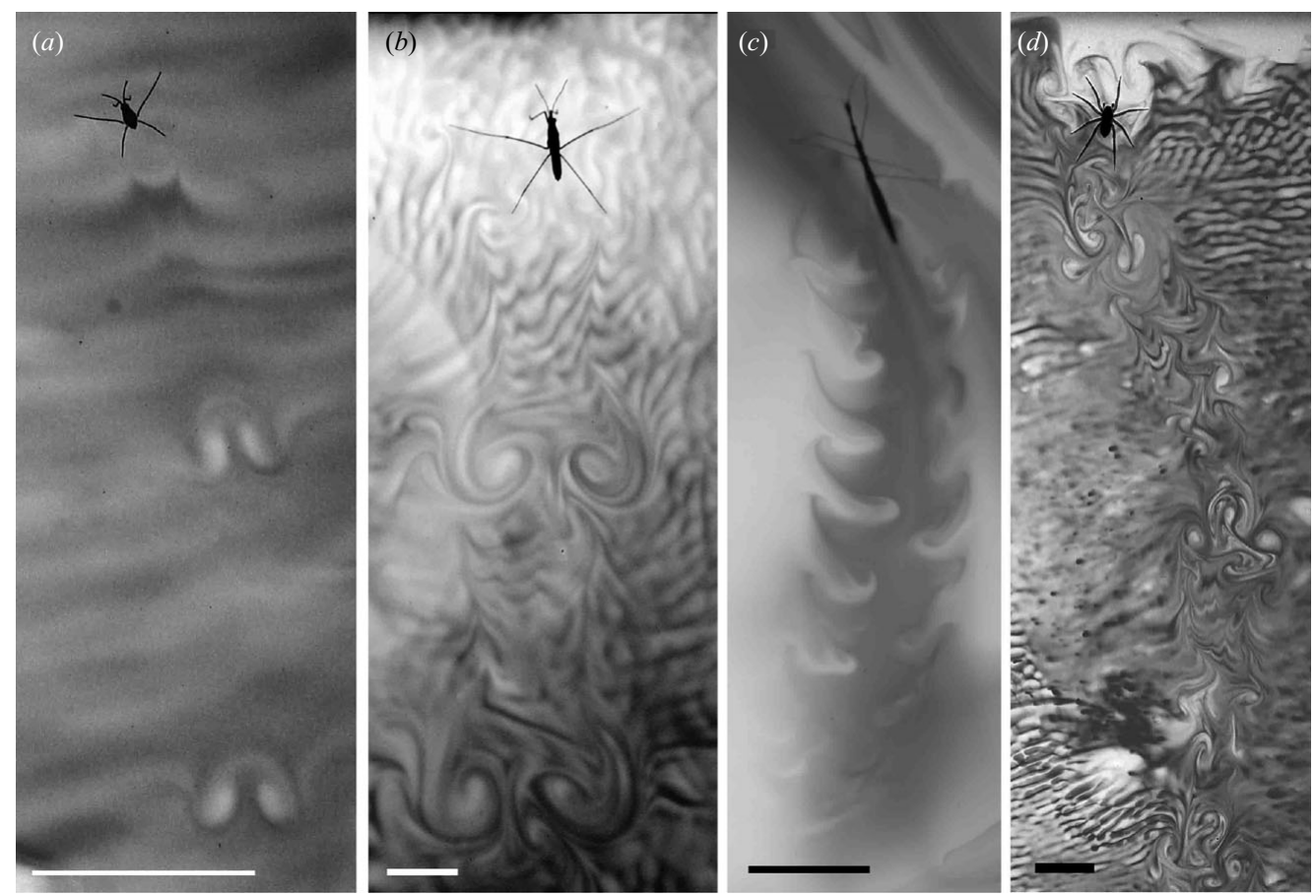

FIGURE 12. Vortices in the wake of water walkers, as visualized by Thymol Blue: $(a)$ the infant water strider, $(b)$ the adult water strider, $(c)$ Hydrometra, $(d)$ the fisher spider. Each leg stroke generates a vortex. Scale bars, $1 \mathrm{~cm}$.

free surface (figure 13a,b), generating macroscopic curvature forces and microscopic contact and viscous forces. It is noteworthy that the infant water strider generates almost no surface deflection but instead appears to brush the free surface (figure $14 a$ ). Flow visualization of the strider wake reveals two salient flow structures, namely capillary waves and vortices (figures $12 a, b$ and 13-15; Hu et al. 2003). These flow structures exist for striders of all sizes, including the infant water strider. The plan 


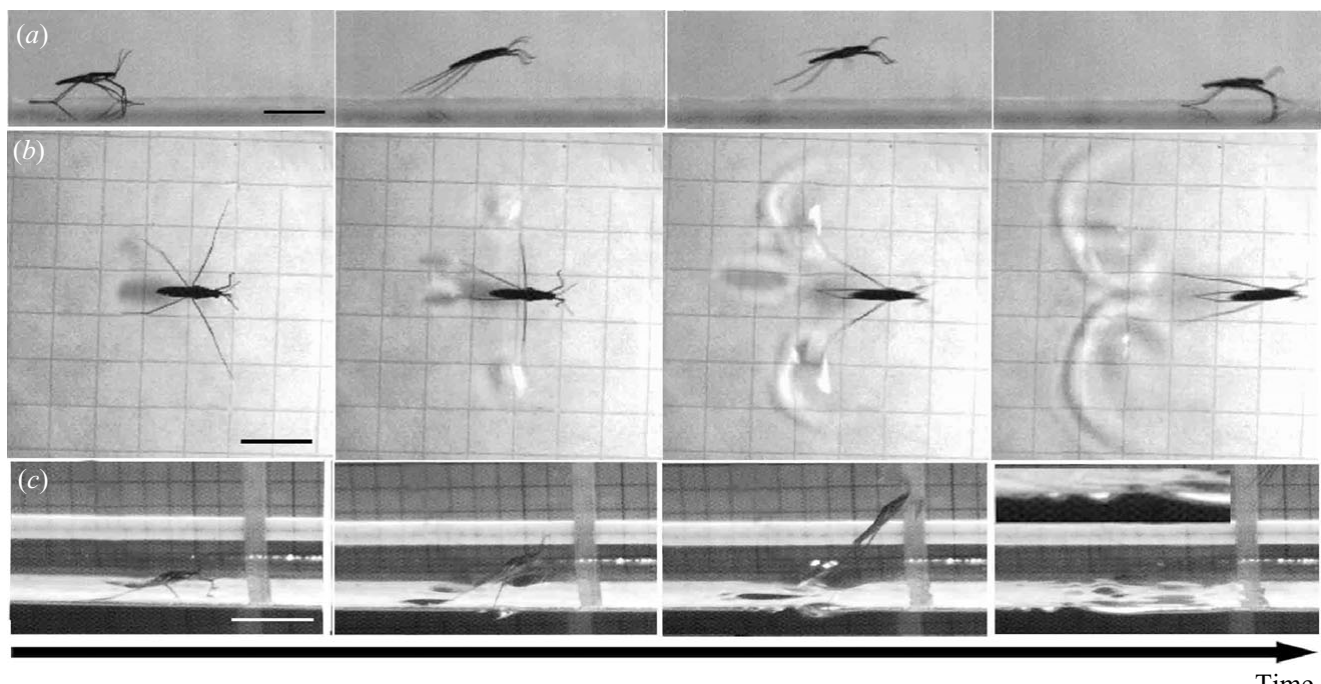

Time

FIGURE 13. The rowing stroke of the adult water strider. Capillary waves are generated by each driving leg and can be seen in magnified view in the inset in $(c)$. The depth by which the free surface is deformed, approximately $2 \mathrm{~mm}$, is evident in (c). Time between frames, $\Delta t=0.01 \mathrm{~s}$. Scale bars, $1 \mathrm{~cm}$
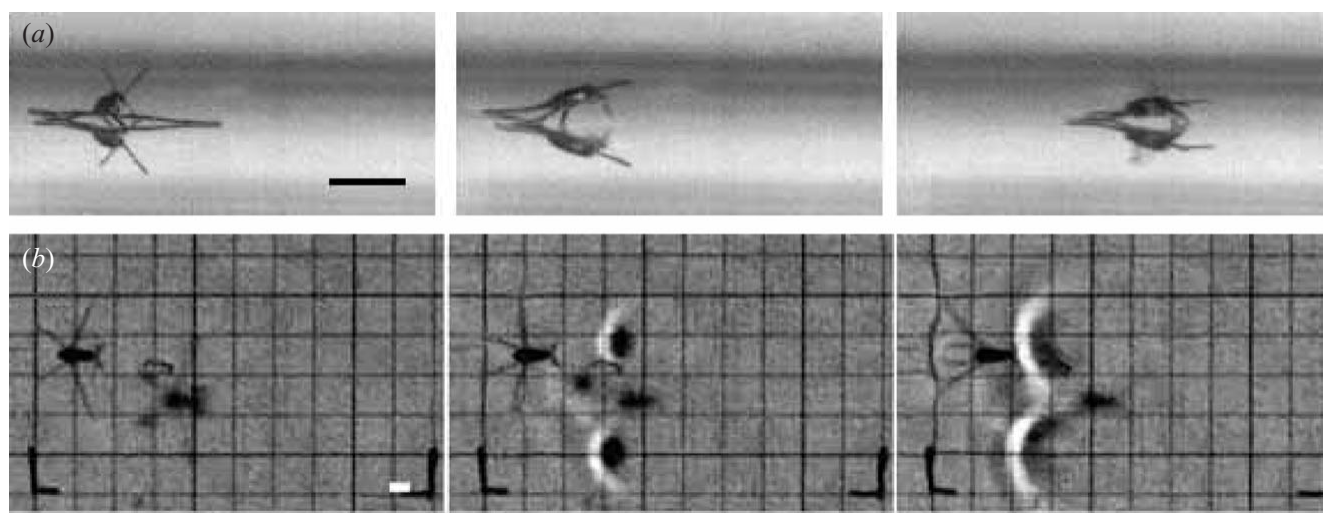

FiguRE 14. (a) An infant water strider propelling itself by brushing the free surface. Very little surface deformation is apparent. As water striders mature and grow, they are capable of higher leg speeds. In $(b)$, an adolescent water strider propels itself with a driving stroke that generates substantial deformation of the free surface. The free surface is lit from above; so the deformation casts a shadow. Time between frames: (a) 1/500 s, (b) 3/500 s. Scale bars, $1 \mathrm{~mm}$.

view in figure 14(b) shows the rearward propagation of a wave generated by the rowing stroke. In plan view, capillary waves are marked by dark and light regions on the bottom of the tank associated with the diffraction of light by the crests and troughs of the wave, respectively.

The generation of subsurface vortices is shown in figure 15 , in which a water strider rows across a dyed front of Thymol Blue. The water strider begins at rest on the free surface, with the surface deforming to a depth of order $h \sim 0.1 \mathrm{~cm}$ (Matsuda, Watanabe \& Eiju 1985). The water strider swings its driving legs in an arc over a duration of $0.01 \mathrm{~s}$. With this leg stroke, it generates a jet that entrains dyed fluid 

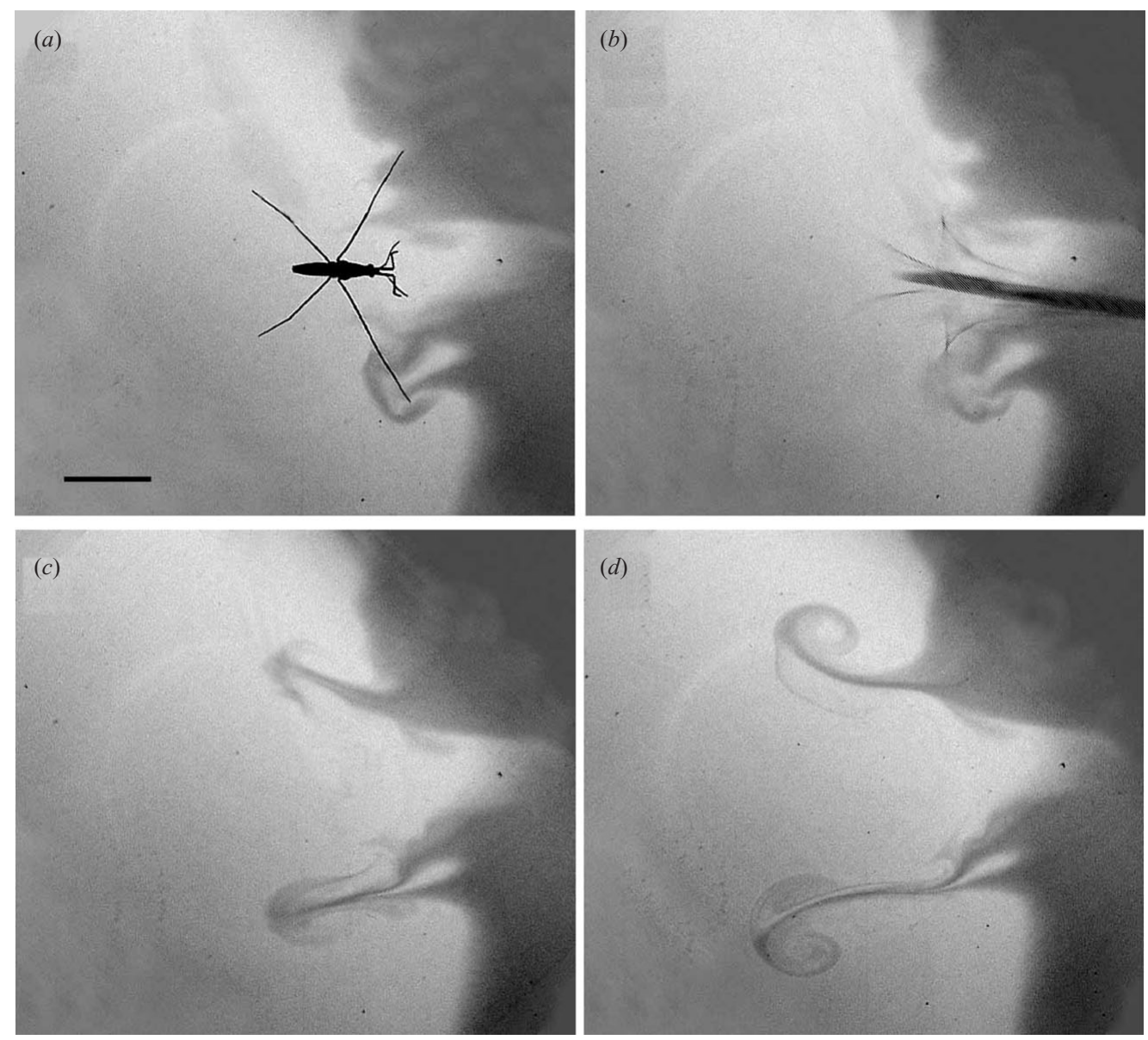

FIGURE 15. Vortex generation by the water strider. The strider rows its legs across a section of dyed fluid. The rowing stroke generates a pair of jets that roll up into a pair of dyed dipolar vortices. Time sequence of frames: $[0,0.03,0.2,0.8 \mathrm{~s}]$. Scale bar, $1 \mathrm{~cm}$. Seconds after their production, the hemispherical vortices are slowed to a halt by viscous forces.

and rolls up into a vortex that translates backward at a characteristic initial speed of $4 \mathrm{~cm} \mathrm{~s}^{-1}$ before spinning down. Figure 12 $(a, b)$ illustrates the vortical wakes of adult and infant water striders.

Another creature that uses the rowing gait is the fisher spider Dolomedes that rows with its front-most three pairs of legs. The spider supports its weight using its remaining legs and underbelly (figure $16 a, b$ ). Side views of the rowing stroke indicate that, as with the water strider, the leg is swept downward and backward, generating a meniscus approximately $2 \mathrm{~mm}$ in depth. Unlike the water strider, the fisher spider is rarely airborne after rowing. The flow visualization in figure $12(d)$ shows that the spider generates a dipolar vortex with each leg stroke. We note that the vortices interfere, obscuring their form.

To achieve the peak speeds needed to escape predators, fisher spiders transition from rowing to galloping (Suter et al. 1997; Suter \& Wildman 1999). While the spider rows with straight legs, it gallops by bending its legs and striking them downward vertically (figure $16 c, d$ ). Side views of the leg stroke (figure 16c) indicate that the 

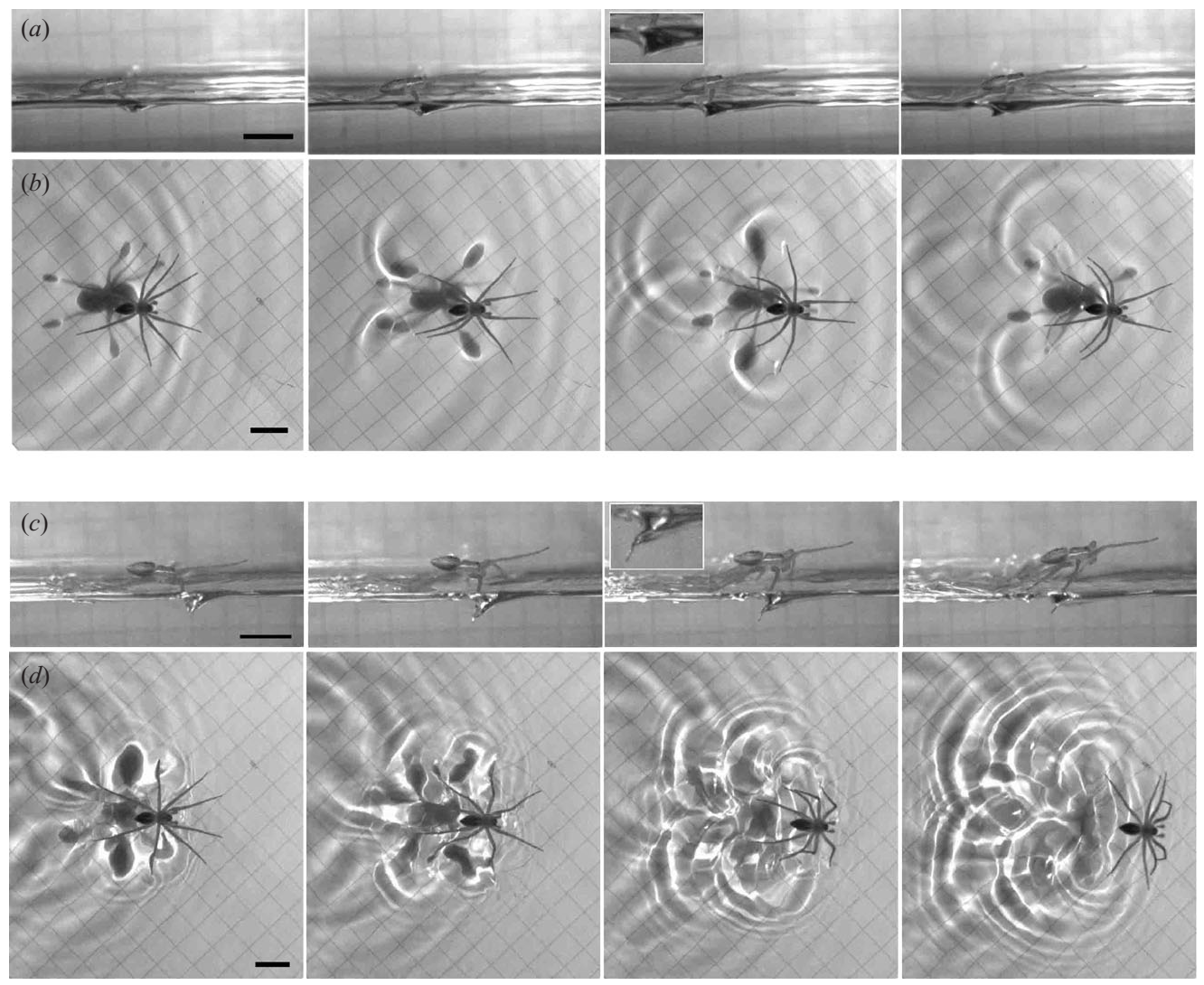

FiguRE 16. $(a, b)$ Rowing and $(c, d)$ galloping by the fisher spider. At body speeds less than $20 \mathrm{~cm} \mathrm{~s}^{-1}$, it rows by driving its legs horizontally along the water surface (Suter et al. 1997). In order to achieve higher speeds, the spider drives its legs vertically to gallop across the water surface. $(b, d)$ The deformation of the free surface is reflected in the shadows cast by the menisci. The insets provide magnified views of the driving legs. For side views, $\Delta t=1 / 200 \mathrm{~s}$; for plan views, $\Delta t=3 / 200 \mathrm{~s}$. Scale bars, $7 \mathrm{~mm}$.

legs generate a meniscus nearly $4 \mathrm{~mm}$ in depth. The advantage of striking the leg vertically is that the spider increases the time during which it is airborne, thereby decreasing the drag experienced in gliding along the interface. Note that while the leg tips penetrate the free surface, their silvery sheen indicates that they maintain a thin air layer trapped within their integument. They thus retain a Cassie state and so easily withdraw their legs following the driving stroke.

Table 2 lists the Strouhal number $S t=f A / V$, where $f$ and $A$ are the frequency and amplitude of the driving stroke and $V$ is the body speed. We note that rowers (spiders and striders) have the lowest Strouhal numbers $(S t \sim 0.1-0.4)$, while creatures that use an alternating tripod gait have the highest values $(S t \sim 0.5-1.1)$. We further note that Strouhal numbers are also correlated with hair anisotropy (angle of inclination $\phi$, as listed in table 1): insects that employ the alternating tripod gait typically have an isotropic, or non-tilted, integument $\left(\phi \sim 50^{\circ}-90^{\circ}\right)$, while rowers such as the water strider have an anisotropic hair layer $\left(\phi \sim 20^{\circ}-50^{\circ}\right)$. The correlation between anisotropic integument and propulsive efficiency is suggestive of a higher degree of specialization for rowers. 

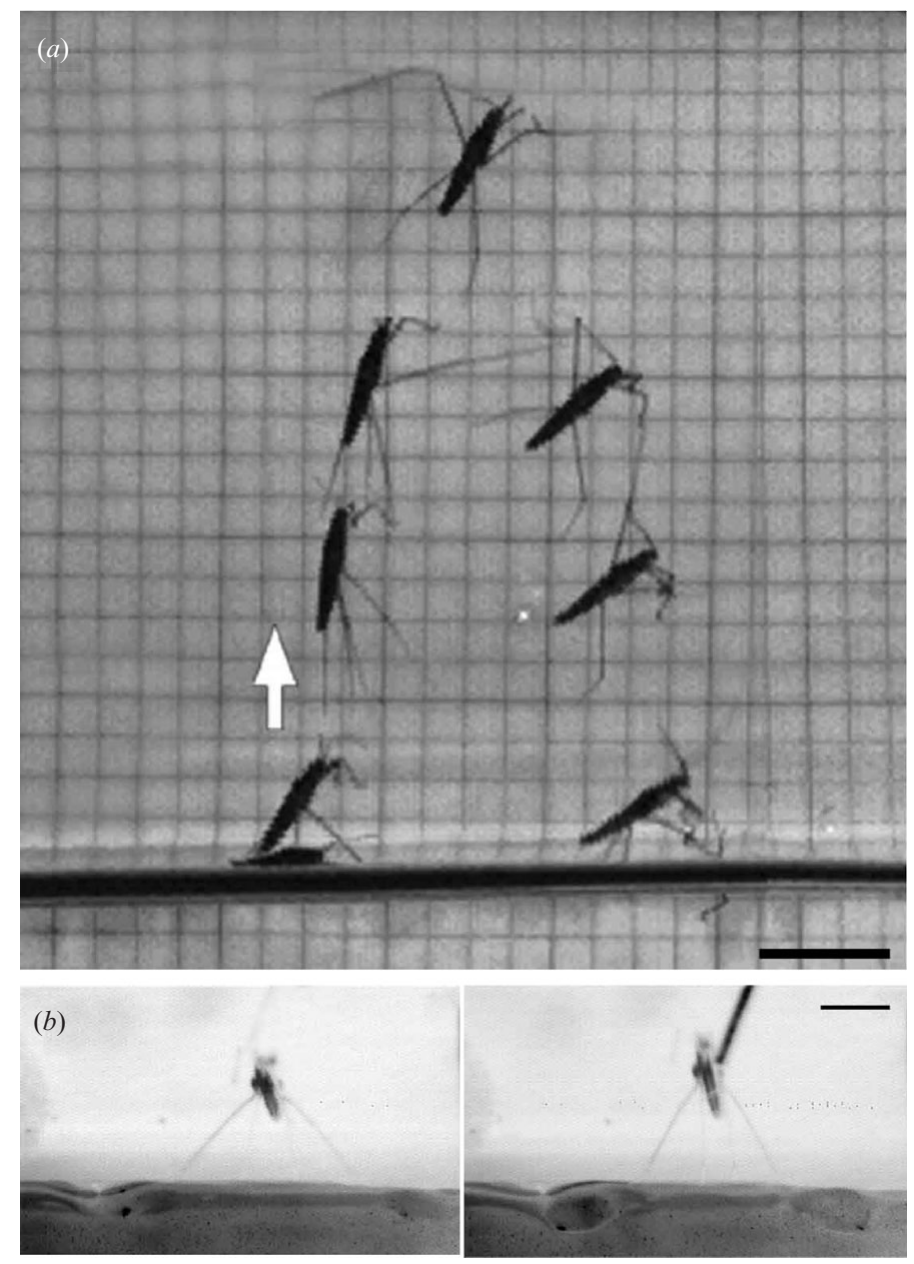

FIGURE 17. The leap of the water strider. (a) Superimposed video frames show the trajectory of the leap. Take-off is accomplished by the strider driving its middle legs vertically against the free surface. $(b)$ Front view of the fluid disturbance generated by the leap, visualized using Kalliroscope. Time between video frames $\Delta t=0.004 \mathrm{~s}$. Scale bars, $1 \mathrm{~cm}$.

A number of water-walking arthropods, including water striders, spiders and Collembola, can leap off the free surface using a variety of techniques (Suter \& Gruenwald 2000; Suter 2003). The strider can row so vigorously that it rockets into the air and lands more than 10 body lengths away (figure 13c). To leap, the strider simply modifies the impact angle of its driving stroke (figure 17). After leaping, the striders land with splayed legs, presumably in order to distribute the force of impact over a larger perimeter. On landing, the striders may penetrate the free surface with their front legs, as shown in figure 17(a), but are not wetted by virtue of their integument. During the driving stroke, the meniscus is deformed to a depth of $0.3 \mathrm{~cm}$ (figure 17b). It is noteworthy that their peak leap height on land is comparable to that on water. Fisher spiders can also leap to heights of several body lengths. The spider leaps by driving its eight legs downward simultaneously (figure 18), deforming the free surface to depths of $0.3 \mathrm{~cm}$ (see the inset in figure 18). The spider lands on its underbelly and splayed legs. 

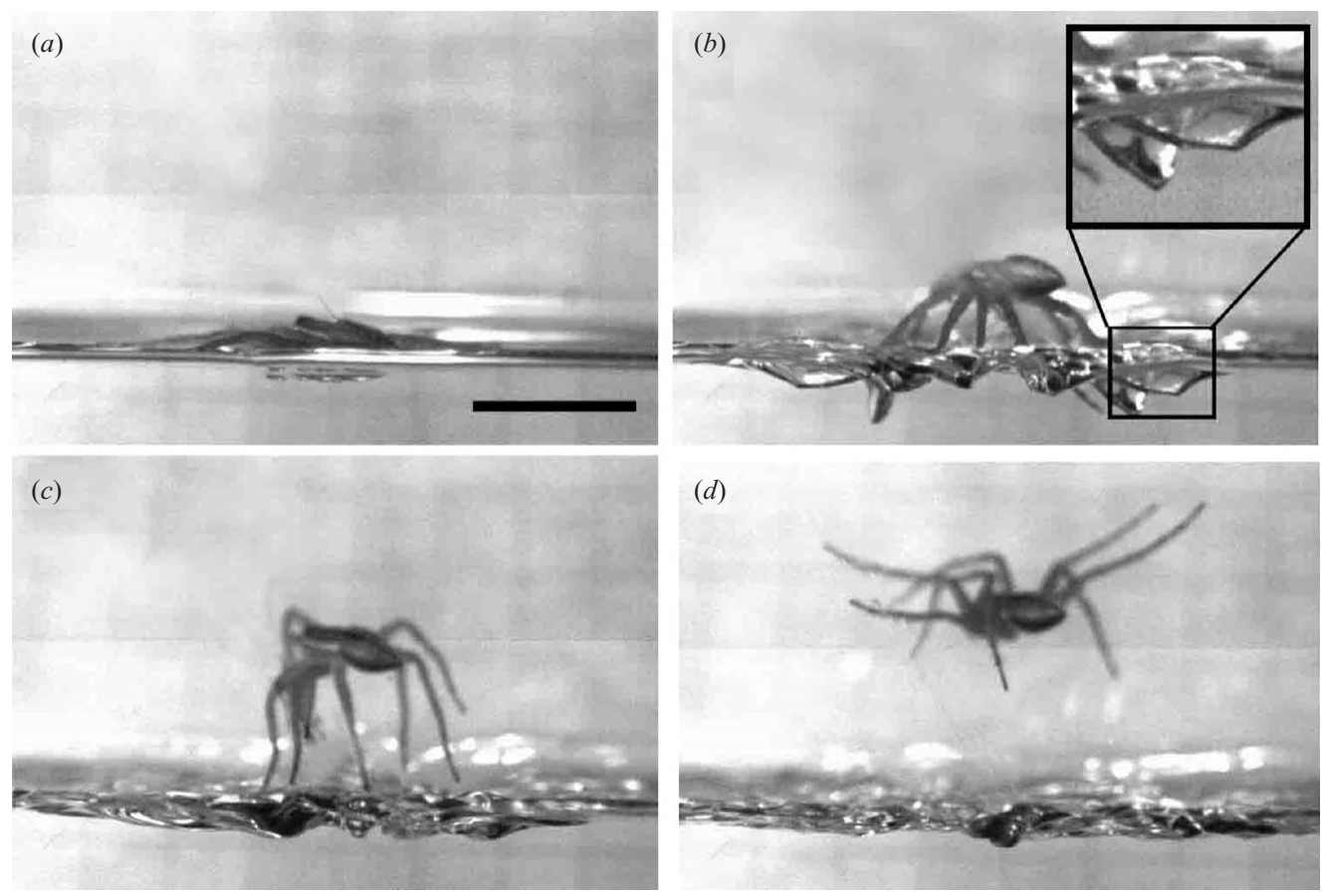

FIGURE 18. The leap of the fisher spider. Take-off follows the spider driving its legs downward. At the apex of its leap, the spider splays its legs outward in preparation for landing. Time between frames, $\Delta t=0.0125 \mathrm{~s}$. Scale bars, $7 \mathrm{~mm}$.

\section{Theoretical developments}

We proceed by developing a theoretical description of the interaction of a waterwalking arthropod with the interface and underlying fluid. The force balance in static and dynamic states is considered in $\S 5.1$, and the momentum transfer across the interface is characterized in $\S 5.2$.

\subsection{Force balance}

We model an object of mass $M$ and speed $\boldsymbol{U}$ with contact line $C$ and a wetted contact area $S_{b}$ (figure 19). The fluid of density $\rho$ and viscosity $\mu$ is characterized by a stress tensor $\boldsymbol{T}=-p \boldsymbol{I}+2 \mu \boldsymbol{E}$, where $p$ is the fluid pressure, $\boldsymbol{I}$ the identity matrix and $\boldsymbol{E}$ the rate of strain tensor. The air-water interface has a tension $\sigma$. The rate of change of the momentum of a body is determined by the net force acting on it:

$$
M \dot{\boldsymbol{U}}(t)=\int_{S_{b}} \boldsymbol{T} \cdot \boldsymbol{n} \mathrm{d} S+\int_{C} \sigma \boldsymbol{t} \mathrm{d} \ell+M \boldsymbol{g} .
$$

The pressure can be written as the sum of dynamic and hydrostatic pressures: $p=p_{d}+$ $\rho g z$, where $z$ is the height below the undisturbed water surface. Our experiments indicate that the Reynolds number $R e=(U w) / v$ based on the leg width $w$ and the peak leg speed $U$ characterizing virtually all water walkers is large, and so the pressure may be expressed to leading order by the time-dependent Bernoulli equation $(\partial \phi / \partial t)+(1 / 2)|\boldsymbol{u}|^{2}+(p+\rho g z) / \rho=c$, where $\phi$ is the velocity potential and $c$ a constant. 
(a)
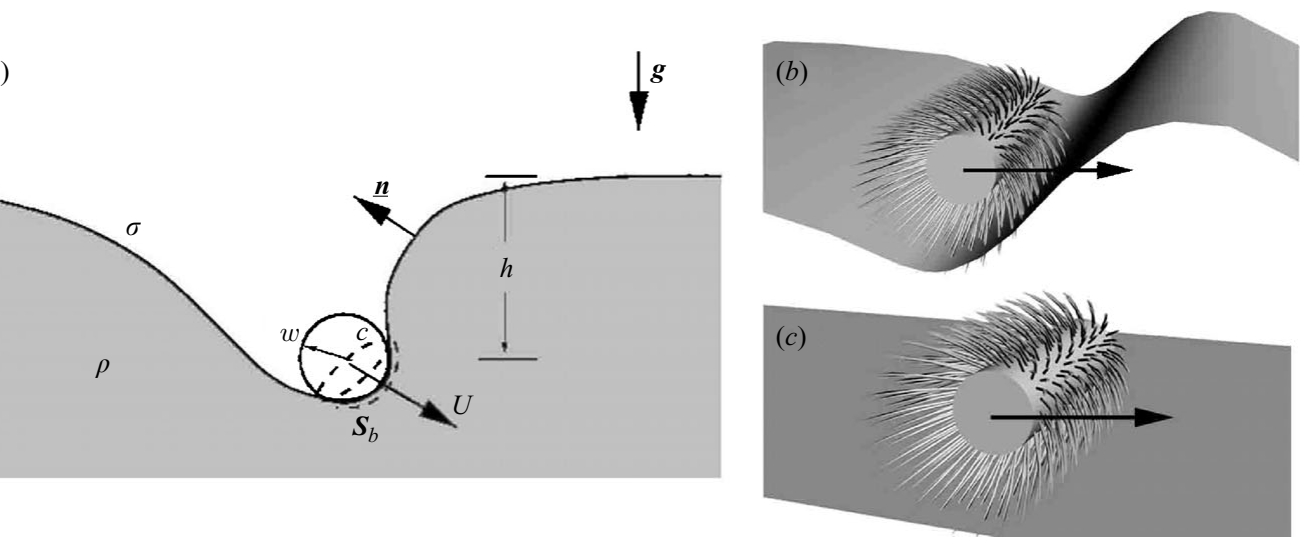

FIGURE 19. (a) A schematic illustration of the driving leg of a water-walking creature. An object of characteristic size $w$ strikes the free surface at speed $U$. The surface is characterized by a surface tension $\sigma$ and its shape by the unit normal $\boldsymbol{n}$. The object's motion will be resisted by some combination of the forces enumerated in (5.4). A small water walker has two means of propulsion available to it, depending on the speed of its driving legs. If the insect drives its leg at a rate faster than the minimum capillary wave speed $c_{m}$, the free surface will deform asymmetrically around it, as shown in $(a, b)$, generating lateral curvature forces that may be exploited for propulsion. (c) Conversely, if the leg is driven at a rate substantially less than $c_{m}$, the free surface remains quasi-static and so fore-aft symmetric. Propulsion at these speeds relies on the contact or viscous forces generated as the leg brushes the free surface.

Substituting $p$ into the stress tensor $\boldsymbol{T}$ in (5.1) yields

$$
M \dot{\boldsymbol{U}}=M g+\rho \int_{S_{b}} \frac{\partial \phi}{\partial t} \boldsymbol{n} \mathrm{d} S+\int_{S_{b}} \frac{1}{2} \rho u^{2} \boldsymbol{n} \mathrm{d} S+\int_{S_{b}} \rho g z \boldsymbol{n} \mathrm{d} S+2 \mu \int_{S_{b}} \boldsymbol{E} \cdot \boldsymbol{n} \mathrm{d} S+\int_{C} \sigma \boldsymbol{t} \mathrm{d} \ell .
$$

While the viscous flow term (second to last) is negligible when the bulk Reynolds number is large, we include it here in light of our discussion in $\$ 3.2$ of the microhydrodynamics. The above equation shows that the hydrodynamic force may be decomposed into components associated with added mass, form drag, hydrostatic pressure, viscous stress and the surface tension force. We introduce dimensionless variables

$$
u^{\prime} \sim \frac{u}{U}, \quad a^{\prime}=\frac{a}{a_{*}}, \quad x^{\prime}=\frac{x}{w}, \quad z^{\prime}=\frac{z}{h}, \quad \phi^{\prime}=\frac{\phi}{w U},
$$

where $U$ is the characteristic leg speed, $a_{*}$ the leg acceleration, $w$ the leg width and $h$ the mean leg depth. Dividing both sides by $\sigma w$ and dropping the primes yields a dimensionless expression for the total force on the object:

$$
\begin{array}{r}
M \dot{\boldsymbol{U}} / \sigma w=-B a \hat{z}+W e\left(A d \int_{S} \frac{\partial \phi}{\partial t} \boldsymbol{n} \mathrm{d} S+\int_{S_{b}} \frac{u^{2}}{2} \boldsymbol{n} \mathrm{d} S+\frac{1}{R e} \int_{S_{b}} \boldsymbol{n} \cdot \boldsymbol{E} \mathrm{d} S\right) \\
+B o \int_{S_{b}} z \boldsymbol{n} \mathrm{d} S+\int_{C} \boldsymbol{t} \mathrm{d} \ell,
\end{array}
$$

where the dimensionless groups are the Baudoin number $B a=(M g) /(\sigma w)$, Weber number $W e=\left(\rho U^{2} w\right) / \sigma$, Reynolds number $\operatorname{Re}=(w U) / v$, Bond number $B o=(\rho g h w) / \sigma$ and added mass number $A d=\left(V_{d} a_{*}\right) /\left(U^{2} w^{2}\right) ; V_{d}$ is the volume of 
fluid displaced by the driving leg, specifically the volume of the meniscus depression for small water walkers.

Equation (5.4) gives the various components of the hydrodynamic force acting on a water walker's leg. Assessment of the magnitudes of these dimensionless groups in tables 1 and 2 indicates that the great majority of water-walking arthropods depend principally on some combination of curvature forces and form drag for their forward propulsion. However, we have seen in $\S 3.2$ that even in the absence of the strength to distort the interface sufficiently to generate substantial curvature forces, all water walkers have contact and viscous forces available to them for propulsion.

\subsubsection{Weight support}

We proceed by examining the vertical force balance on a water walker. The vertical component of the force balance is given by the dot product of $\hat{z}$ and (5.4). Dividing by the Baudoin number yields

$$
\begin{array}{r}
\frac{\dot{\boldsymbol{U}} \cdot \hat{z}}{g}+\hat{z}=M c\left(A d \int_{S} \frac{\partial \phi}{\partial t} \boldsymbol{n} \cdot \hat{z} \mathrm{~d} S+\int_{S_{b}} \frac{u^{2}}{2} \boldsymbol{n} \cdot \hat{z} \mathrm{~d} S+\frac{1}{R e} \int_{S_{b}} \boldsymbol{n} \cdot \boldsymbol{E} \cdot \hat{z} \mathrm{~d} S\right) \\
+\frac{B o}{B a} \int_{S_{b}} z \boldsymbol{n} \cdot \hat{z} \mathrm{~d} S+\frac{1}{B a} \int_{C} \boldsymbol{t} \cdot \hat{z} \mathrm{~d} \ell,
\end{array}
$$

where we have introduced the McMahon number $M c=\left(\rho U^{2} w^{2}\right) /(M g)$. An object with zero initial vertical speed can be supported at the interface if the time average of $\dot{\boldsymbol{U}} \cdot \hat{z}$ is zero. We consider creatures in steady-state locomotion, for which this latter condition automatically holds.

First, (5.5) indicates that small creatures $(B a=(M g) /(\sigma w)<1)$ may reside at rest $(M c=0)$ at the free surface. For creatures too massive to rely on surface tension for weight support $(B a \gg 1),(5.5)$ indicates two means of walking on water. If the creature has a mean density less than that of water, as is the case for many aquatic fowl such as ducks, its weight may be supported by flotation. However most creatures have a density slightly in excess of that of water. Such heavy creatures can support their weight in a dynamic state if $M c>1$, as is the case for basilisk lizards (Glasheen \& McMahon 1996a,b).

In a static situation $(M c=0),(5.5)$ yields a generalized form of the Archimedes principle. The force on a static floating body is equal to the weight of the fluid displaced:

$$
M \boldsymbol{g} \cdot \hat{z}=\boldsymbol{F}_{\boldsymbol{b}} \cdot \hat{z}+\boldsymbol{F}_{\boldsymbol{c}} \cdot \hat{z}=\int_{S_{b}} \rho g z \boldsymbol{n} \cdot \hat{z} \mathrm{~d} s+\sigma \int_{C} \boldsymbol{t} \cdot \hat{z} d \ell=\rho g V_{b}+\rho g V_{m} .
$$

Mansfield et al. (1997) and Keller (1998) showed that the magnitudes of the buoyancy and curvature forces on a floating body are equal to the weights of the fluid displaced by the meniscus, respectively, inside and outside the line of tangency $C$ (respectively $V_{b}$ and $V_{m}$ in figure 20b). For long thin bodies such as water-walking insect legs, the ratio of buoyancy to curvature forces is thus given by the ratio of the leg radius $w$ to the capillary length $\ell_{c}=\sqrt{\sigma /(\rho g)}$ or, equivalently, the square root of the Bond number:

$$
\frac{F_{b}}{F_{c}} \sim \frac{V_{b}}{V_{m}} \sim \frac{w}{\ell_{c}} \sim \sqrt{B o} .
$$




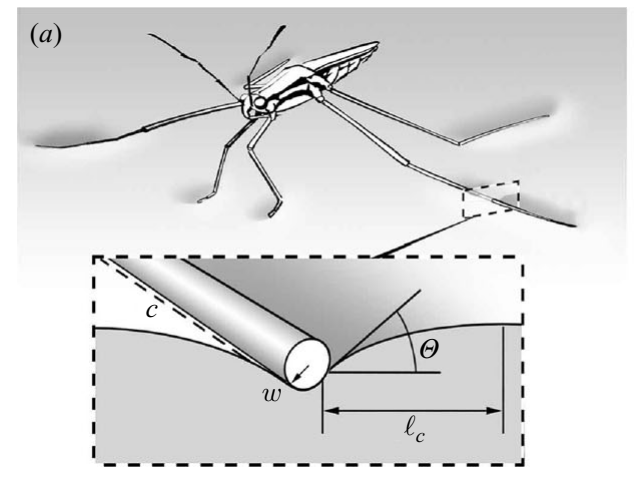

(b)

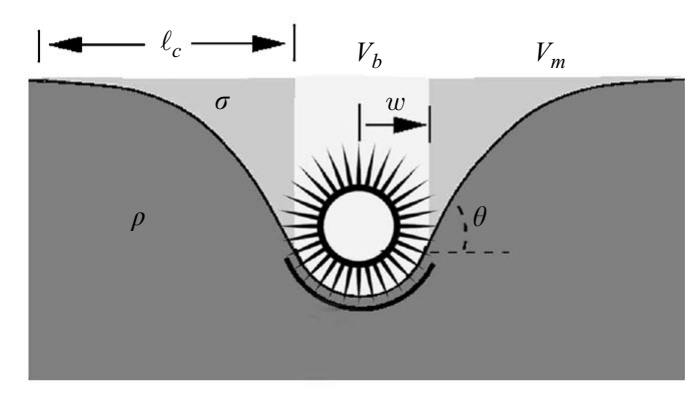

FIGURE 20. The static weight support of the water strider. (a) The weight of water-walking arthropods is supported by curvature forces associated with deformations of the free surface. The leg of width $w$ intersects the free surface at a contact line $C$. The initial angle of tangency between the free surface and the horizontal is $\theta$ : the resulting meniscus decays over the capillary length $\ell_{c}$. (b) The leg in cross-section; $V_{b}$ and $V_{m}$ denote the volume of fluid displaced inside and outside the contact line, respectively. As shown, the hairiness of the leg effectively increases its volume without substantially increasing its mass, thus contributing to its buoyancy.

The characteristic Bond numbers of the water-walking insects considered in our study are listed in table 2 . Water striders (with typical weight 3-10 dynes and leg width $20-80 \mu \mathrm{m}$ ) have $10^{-4}<B o<10^{-2}$ and are thus supported almost exclusively by surface tension. The relatively stocky fisher spider (weight $10^{2}-10^{3}$ dynes and leg width up to $0.17 \mathrm{~cm}$ ) has $10^{-3}<B o<10^{-1}$ and so may have up to one third of its weight supported by buoyancy. Finally we note that an insect augments its buoyancy with the air layer trapped in its integument, which may increase the volume of fluid displaced by $20-30 \%$ (Bush et al. 2008).

\subsubsection{Lateral propulsion}

The horizontal component of the force balance on a water walker is given by the $\hat{x}$ component of (5.4):

$$
M \dot{\boldsymbol{U}} \cdot \hat{x} /(\sigma w)=W e\left(A d \int_{S_{b}} \frac{\partial \phi}{\partial t} \boldsymbol{n} \cdot \hat{x} \mathrm{~d} S+\int_{S_{b}} \boldsymbol{n} \cdot \hat{x} \mathrm{~d} S\right)+B o \int_{S_{b}} z \boldsymbol{n} \cdot \hat{x} \mathrm{~d} S+\int_{C} \boldsymbol{t} \cdot \hat{x} \mathrm{~d} \ell .
$$

For large creatures $(W e \gg 1$ and $B o \gg 1)$, surface tension is negligible, and (5.8) shows that such water-walking creatures may propel themselves using inertial and hydrostatic pressures. We note that the hydrostatic pressures can only produce a lateral force on a body with a cavity that is not fore-aft symmetric. For example, the basilisk lizard generates thrust using hydrostatic pressure by generating an air cavity with its feet and pressing against the cavity's back wall (Glasheen \& McMahon $1996 a)$.

In the parameter regime of most water-walking arthropods, $(W e, B o) \ll 1$, surface tension dominates both inertial and hydrostatic forces, so that

$$
M \dot{U} \cdot \hat{x} /(\sigma w)=\int_{C} \boldsymbol{t} \cdot \hat{x} \mathrm{~d} \ell .
$$

The dominant lateral propulsive force comes from the curvature force that may be generated by the driving leg. The interface thus responds roughly like a trampoline. 
The curvature pressure generated by the stroke of a leg of width $w$ is $\sigma / w$; therefore, the net curvature force acting on the driving legs is $F_{\sigma}=A \sigma / w$, where $A$ is the area of the driving leg adjoining the distorted interface. We note that such a force can only be effectively generated if the speed of the leg strike exceeds the capillary wave speed; otherwise, the interface will respond in a quasi-static manner, assuming a fore-aft symmetric form incapable of propelling the creature forward with curvature forces. At such low speeds, the creature must rely on the brushing technique, in which the propulsive force has its origins in the microscopic contact forces or viscous stresses acting on the wetted hair tips on the driving leg.

By considering the leg strike on a macroscopic scale, we have deduced an estimate for the curvature force $F_{\sigma}$ generated by the driving stroke. In (3.2), we deduced an estimate for the brushing force $F_{b r u s h}=n A F_{\text {contact }}^{\|}$associated with the microstructure on the driving legs. The relative magnitudes of these two forces acting on a leg area with hair density $n$ and width $w$ are given by

$$
\frac{F_{\text {brush }}}{F_{\sigma}}=\frac{n \sigma L A \Delta \cos \theta}{A \sigma / w}=n w L \Delta \cos \theta .
$$

The magnitude of this ratio depends on the magnitude of the contact angle hysteresis, but it is noteworthy that the brushing force resulting from the microscale interaction between integument and interface may be comparable to the curvature forces. For the physical variables listed in table 2, this ratio assumes values between 0.1 and 1 , suggesting the dominance of macroscopic curvature pressures in the propulsive force. However, situations arise in which water-walking arthropods are too small, slow and weak to generate substantial surface distortions and propulsive curvature forces. In such situations, the creatures are still able to move by brushing the free surface.

We proceed by considering the hypothetical case of a creature jumping on a soap film. Note that this is but a theoretical abstraction owing to the fact that soap destroys the water repellency of insect legs, causing them to puncture and break the film. In the relevant ( $W e, B o \ll 1)$ limit, the force balance on the creature, (5.1), assumes the form

$$
M \dot{\boldsymbol{U}} \cdot \hat{z}=\int_{C} \sigma \boldsymbol{t} \cdot \hat{z} \mathrm{~d} \ell+M g .
$$

The only forces acting on the creature are those that are due to surface tension and gravity. The creature is static $(\dot{\boldsymbol{U}}=0)$ when its weight is balanced by the curvature force associated with deformation of the free surface. A lateral propulsive force is possible only if the meniscus is distorted asymmetrically; however, such a distortion requires that the leg strikes the film at a speed exceeding the capillary wave speed. On a soap film of thickness $h \sim 4 \mu \mathrm{m}$, this wave speed is approximately $\sqrt{2 \sigma / \rho h} \sim 5 \mathrm{~m} \mathrm{~s}^{-1}$, well beyond the peak leg speed of any water walker. Consequently, water-walking arthropods could not propel themselves via curvature forces on a soap film: the absence of inertia of the underlying fluid precludes their principle mode of propulsion. We note that brushing forces might yet allow motion on a soap film, as they do for slow water walkers at the water surface. While this is but a theoretical abstraction, it underscores the subtle interplay of wetting properties, waves and momentum transfer that lies at the heart of this style of biolocomotion.

\subsection{Momentum transfer}

Having considered the hydrodynamic force on an object striking the free surface, we here consider the transfer of momentum by the object into the control volume 


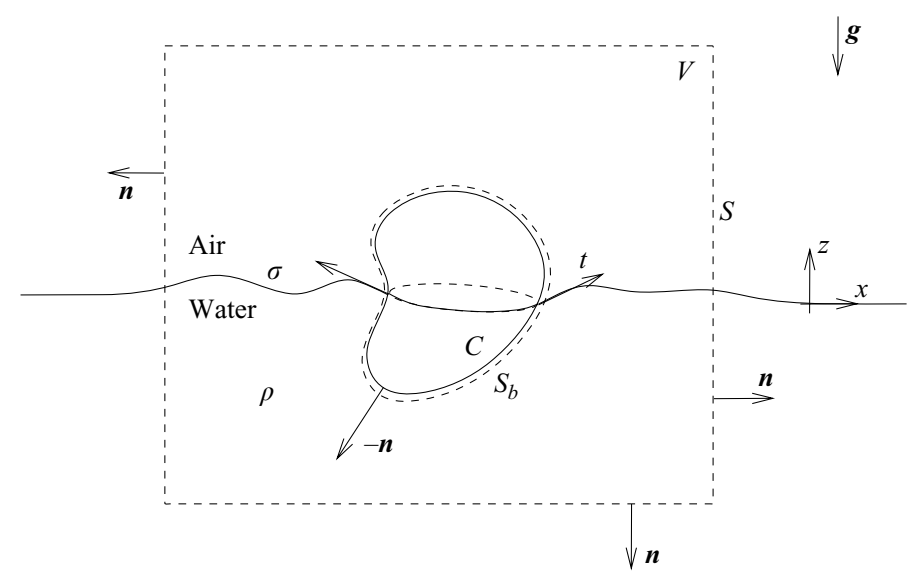

FIGURE 21. A body striking the free surface. The control volume $V$ is bounded outside by $S$ and inside by the body surface $S_{b}$. The body intersects the free surface at a contact line $C$; $\boldsymbol{n}$ denotes the outward unit normal to the fluid bound by $S_{b}$.

illustrated in figure 21. Our analysis builds upon the framework developed by Childress (1981) to describe swimmers and fliers to incorporate the influence of the interface on propulsion. From first principles, we may write the conservation of momentum in the fluid as

$$
\frac{\partial}{\partial t} \int_{V} \rho \boldsymbol{u} \mathrm{d} V=-\int_{S}(\rho \boldsymbol{u} \boldsymbol{u} \cdot \boldsymbol{n}+p \boldsymbol{n}) \mathrm{d} S+\int_{S_{b}} \boldsymbol{t}_{a p p} \mathrm{~d} S+\int_{V} \rho \boldsymbol{g} \mathrm{d} V,
$$

where $\boldsymbol{t}_{a p p}$ is the local stress applied by the object to the fluid and $S_{b}$ is the wetted body surface. Newton's third law requires that

$$
-\int_{S_{b}} \boldsymbol{t}_{a p p} \mathrm{~d} S=\int_{S_{b}} \boldsymbol{T} \cdot \boldsymbol{n} \mathrm{d} S+\int_{C} \sigma \boldsymbol{t} \mathrm{d} \ell .
$$

The sum of (5.1), (5.12) and (5.13) yields

$$
M \dot{\boldsymbol{U}}+\frac{\partial}{\partial t} \int_{V} \rho \boldsymbol{u} \mathrm{d} V=-\int_{S}(\rho \boldsymbol{u} \boldsymbol{u} \cdot \boldsymbol{n}+p \boldsymbol{n}) \mathrm{d} S+M g \hat{z}-\rho g\left(V_{m}+V_{b}\right) \hat{z},
$$

where $V_{m}+V_{b}$ is the net fluid volume displaced by the object (figure 20). We note that this reduces to the generalized form of the Archimedes principle (5.6) in the static case. It also indicates that creatures too heavy to float at the surface can only remain there by generating a vertical flux of fluid. One may thus rationalize the vertical component of the vortices shed by the driving stroke of the basilisk lizard (Hsieh 2003, 2004). Conversely, water-walking arthropods rely on surface tension for weight support but require the horizontal transfer of momentum for their lateral propulsion.

Equation (5.14) is the statement of conservation of momentum for an object at a free surface: the creation of horizontal momentum in the fluid by the driving stroke of a water walker indicates a lateral force on the creature. Note that surface tension does not arise in (5.14): while it contributes a force to both the body and the control volume, these contributions are equal and opposite. Of course, the absence of surface tension in the momentum balance may also be simply understood on the grounds that 
the interface carries no mass. As for swimming fish (Wilga \& Lauder 2002) and flying birds (Spedding et al. 2003), one may rationalize the propulsion of water-walking creatures by considering the momentum transferred in their wake (Dickinson 2003; Dabiri 2005).

\section{Discussion}

The dynamics of water-walking arthropods was previously described (Denny 1993; Vogel 1994) by treating their legs as smooth cylinders: macroscopic curvature forces were generated as the interface responded as a trampoline to the driving stroke. We have demonstrated here that propulsion at the interface is instead a rich, multiscale problem in which the micron-scale roughness of the integument plays a critical role. Over the range of hydrostatic and dynamic pressures experienced by water walkers, their integument maintains a Cassie state. The integument couples with the underlying fluid on the microscale through combined viscous and contact forces, the relative magnitudes of which depend on the geometry of the integument. The notion of contact forces is useful for rationalizing several previously enigmatic behaviours of water walkers. Larger water walkers can strike the surface at a speed exceeding the capillary wave speed, thus generating an asymmetric meniscus and the concomitant macroscopic curvature forces. Conversely, smaller, weaker water walkers generate negligible surface deformation during their stroke and so rely principally on microscale contact and viscous forces generated by the leg brushing the free surface.

We have demonstrated the utility of the Strouhal number, a common measure of dynamic efficiency in other modes of biolocomotion, for characterizing propulsion on the water surface. The Strouhal number is the ratio of a creature's peak appendage speed to its body speed. By virtue of their solid contact with the ground, terrestrial creatures have Strouhal numbers near 1. Efficient swimmers and fliers tend to have Strouhal numbers of $0.2-0.4$, as shown in the studies of Taylor, Nudds \& Thomas (2003) and Alexander (2003). For water walkers, for which $0.1<S t<0.4$, thrust is generated by driving against water, while drag is experienced in air, at least during their airborne phase. Thus, from a Strouhal number perspective, rowing on water is of comparable efficiency to swimming and flying.

In our experimental investigation, we have reported the prevalence of coherent vortices in the wake of water-walking arthropods. The propulsion mechanism of water-walking insects thus has features in common with swimmers and fliers in that each driving stroke generates a vortex. We developed a theoretical framework that describes the transfer of force and momentum between the water walker and its environment on both the microscopic and the macroscopic scale. Our developments make it clear that momentum is conserved between the fluid and the creature across the interface, an assumption made implicitly by $\mathrm{Hu}$ et al. (2003) and Bühler (2007) in their studies of the water strider and by Hsieh (2004) in her study of the basilisk lizard. The vortical wakes reported by $\mathrm{Hu}$ et al. (2003) and Hsieh (2004) and more comprehensively here may thus be used to simply rationalize the propulsion of water-walking creatures. Our formulation provides an integrative view of all forms of walking on water, including both the inertia-based propulsion of large water walkers and the surface-tension-based propulsion of water-walking arthropods. Moreover, our study informs the resolution of Denny's paradox (Suter et al. 1997).

Denny's paradox (Denny 1993, 2004) was generalized by Suter et al. (1997) to the following form: 'small or slow-moving surface dwelling arthropods should not be able to propel themselves horizontally'. We now see that the paradox rested on 
two flawed assumptions. First, water strider's motion was assumed to rely on the generation of capillary waves, since the propulsive force was thought to be that associated with wave drag on the driving leg. Second, in order to generate capillary waves, it was assumed that the strider leg speed must exceed the minimum wave speed, $c_{m}=(4 g \sigma / \rho)^{1 / 2} \approx 23 \mathrm{~cm} \mathrm{~s}^{-1}$. We note that this second assumption is strictly true only for steady motions (Lighthill 1978) and so is not strictly applicable to the propulsive driving stroke of the water strider; indeed, bodies moving at unsteady speeds $u<c_{\min }=23 \mathrm{~cm} \mathrm{~s}^{-1}$ can produce waves (Dias \& Kharif 1999; Milewski \& Vanden-Broeck 1999; Chepelianskii, Chevy \& Raphäel 2008); bodies moving at steady speeds $u<c_{\min }$ can produce a solitary wave packet with decaying tails (Vanden-Broeck \& Dias 1992; Dias, Menasce \& Vanden-Broeck 1996); and floating bodies oscillating with a vertical speed $U \cos \omega t$ with $U<c_{m}$ may create a wave field (Taneda 1991).

Consideration of the microscale forces acting on the integument of the driving legs makes it clear that water-walking arthropods needn't rely on wave drag, inertial forces or macroscopic curvature forces: contact and viscous forces arising from the interaction of the cuticle and the interface are available for propulsion at any leg speed. Denny's paradox can alternatively be resolved by noting that water-walking creatures transfer momentum to the underlying fluid (Hu et al. 2003). The precise partitioning of momentum between waves and vortices following the impulsive stroke was considered experimentally by Hu et al. (2003) and theoretically by Bühler (2007). Hu et al. (2003) made a rough estimate that the momentum partition between waves and vortices was approximately 1-10. Bühler (2007) deduced that an impulsive forcing at a free surface generates momentum in waves and vortices of relative magnitudes $1 / 3$ and $2 / 3$ respectively. Hsieh $(2003,2004)$ showed that water-running basilisk lizards also generate waves and vortices. The precise partitioning of momentum transfer in waves and vortices in the wake of various water walkers is left as a subject for future consideration.

We have demonstrated that most water-walking arthropods have at their disposal two propulsive forces. If they strike the surface at a speed in excess of the capillary wave speed, $23 \mathrm{~cm} \mathrm{~s}^{-1}$, they can produce a meniscus whose fore-aft asymmetry results in a curvature force that propels them forward. Such is the case for most adult water-walking arthropods, for which the interface serves effectively as a trampoline. Conversely, if the peak speed of the driving leg is substantially less than $23 \mathrm{~cm} \mathrm{~s}^{-1}$, the interface responds quasi-statically; consequently, its fore-aft symmetry is maintained, and no lateral propulsive force results. A number of small and infant water walkers thus use a technique that has not previously been discussed: by brushing their legs across a relatively unperturbed surface, they generate a combination of viscous stresses and contact forces on their wetted integument that serve to propel them forward. We note that the latter brushing technique, inefficient though it is when compared with propulsion via curvature forces, operates at all leg speeds.

Finally, it is noteworthy that we have assumed throughout this study that the insect integument is effectively rigid. An interesting avenue for future research is the role of the integument's elasticity on the water repellency and the dynamics of this class of creatures. Prakash \& Bush (submitted) have demonstrated that the cuticle of water strider's integument is unidirectional by virtue of its elasticity: contact forces acting on moving droplets are greatest for motion perpendicular to the leg and smallest for motion towards the leg tip. This observation raises a number of interesting dynamical questions, including the role of the integument in detaching from the free surface. This class of problems is currently under consideration and is likely to inform the design of biomimetic water-walking devices (Hu et al. 2003, 2007; Suhr et al. 2005; 
Floyd et al. 2006; Song, Suhr \& Sitti 2006; Yu et al. 2007) and synthetic unidirectional superhydrophobic surfaces (Prakash \& Bush, submitted).

Video images of many of our experiments can be found at http://www.me. gatech.edu/hu/ or on the Multimedia Fluid Mechanics CD-ROM (Bush \& $\mathrm{Hu}$ 2004).

The authors thank Manu Prakash for many valuable discussions and his contributions to the figures and Lucy Mendel and Brian Chan for their assistance with illustrations. The authors gratefully acknowledge the financial support of the NSF: J. B. through grant CTS-0624830 and Career Grant CTS-0130465 and D. H. through a Mathematical Sciences Postdoctoral Research Fellowship.

\section{REFERENCES}

Aldrovandi, U. 1618 Historiam naturalem de Animalibus Insectis Libri Septem. Typis Pauli Jacobi.

Alexander, R. M. 2003 Principles of Animal Locomotion. Princeton University Press.

Altendorfer, R., Moore, N., Komsuoglu, H., Buehler, M., Brown, H. B. Jr., McMordie, D., Saranli, U., Full, R. \& KoditscheK, D. E. 2001 RHex: a biologically inspired hexapod runner. J. Auton. Robots 11, 207-213.

ANDERSEN, N. M. 1976 A comparative study of locomotion on the water surface in semiaquatic bugs (insects, Hemiptera, Gerromorpha). Vidensk. Meddr. Dansk. Naturh. Foren. 139, 337-396.

ANDERSEN, N. M. 1977 Fine structure of the body hair layers and morphology of the spiracles of semiaquatic bugs in relation to life on the water surface. Vidensk. Meddr. Dansk. Naturh. Foren. 140, 7-37.

Bartolo, D., Bouamrirene, F., Verneuil, E., Beguin, A., Silberzan, P. \& Moulinet, S. 2006 Bouncing or sticky droplets: impalement transitions on superhydrophobic micropatterned surfaces. Europhys. Lett. 74, 299-305.

Baudoin, R. 1955 La physico-chimie des surfaces dans la vie des Arthropodes aeriens des miroirs d'eau, des rivages marins et lacustres et de la zone intercotidale. Bull. Biol. Fr. Belg. 89, $16-164$.

Bowdan, E. 1978 Walking and rowing in the water strider, Gerris remigis. J. Compar. Physiol. $123,43-49$.

Brocher, F. 1910 Les phénomènes capillaires, leur importance dans la biologie aquatique. Ann. Biol. Lacustre 4, 89-139.

BüHLER, O. 2007 Impulsive fluid forcing and water strider locomotion. J. Fluid. Mech. 573, 211-236.

Bush, J. W. M. \& Hu, D. L. 2004 Walking on water. In Multimedia Fluid Mechanics CD (ed. G. M. Homsy). Cambridge University Press.

Bush, J. W. M. \& Hu, D. L. 2006 Walking on water: biolocomotion at the interface. Annu. Rev. Fluid Mech. 38, 339-369.

Bush, J. W. M., Hu, D. L. \& Prakash, M. 2008 The integument of water-walking arthropods: form and function. Adv. Insect Physiol. 34, 117-192.

Cassie, A. B. D. \& BaXter, S. 1944 Wettability of porous surfaces. Trans. Faraday Soc. 40, 546-551.

Chepelianskit, A. D., Chevy, F. \& Raphëel, E. 2008 On capillary-gravity waves generated by a slowly moving object. Phys. Rev. Lett. p. 074504.

Childress, S. 1981 Mechanics of Swimming and Flying. Cambridge University Press.

DABIRI, J. O. 2005 On the estimation of swimming and flying forces from wake measurements. J. Exp. Biol. 208, 3519-3532.

DARnhOFER-Demar, B. 1969 Zur fortbewegung des wasserläufers Gerris lacustris L. auf des wasseroberfläche. Zool. Anz. Suppl. 32, 430-439.

Denny, M. W. 1993 Air and Water: The Biology and Physics of Life's Media. Princeton University Press.

Denny, M. W. 2004 Paradox lost: answers and questions about walking on water. J. Exp. Biol. 207, 1601-1606.

Dias, F. \& KHARIF, C. 1999 Numerical study of capillary-gravity solitary waves. Annu. Rev. Fluid Mech. 31, 301-346. 
Dias, F., Menasce, D. \& Vanden-Broeck, J. M. 1996 Numerical study of capillary-gravity solitary waves. Eur. J. Mech. B 15, 17-36.

Dickinson, M. H. 2003 Animal locomotion: how to walk on water. Nature 424, 621-622.

Dufour, L. 1833 Recherches Anatomiques et Physiologiques sur les Hémiptères, Accompagnées de Considèrations Relatives à l'Histoire Naturelle et à la Classification de ces Insectes, pp. 68-74. Impr. de Bachelier, extrait des Mémoires des savants étrangers, tome IV.

Dussan, E. B. 1979 On the spreading of liquids on solid surfaces: static and dynamic contact lines. Annu. Rev. Fluid Mech 11, 371-400.

Dussan, E. B. \& Chow, R. T. 1983 On the ability of drops or bubbles to stick to non-horizontal surfaces of solids. J. Fluid Mech. 137, 1-29.

Floyd, S., Keegan, T., Palmisano, J. \& Sitti, M. 2006 A novel water running robot inspired by basilisk lizards. In Proceedings of the IEEE/RSJ International Conference on Intelligent Robots and Systems, pp. 5430-5436.

FLYNN, M. R. \& BUSH, J. W. M. 2008 Underwater breathing: the mechanics of plastron respiration. J. Fluid Mech. 608, 275-296.

GAO, X. \& JiANG, L. 2004 Water-repellent legs of water striders. Nature 432, 36.

de Gennes, P. G., Brochard-Wyart, F. \& Quéré, D. 2003 Capillarity and Wetting Phenomena: Drops, Bubbles, Pearls and Waves. Springer.

Glasheen, J. W. \& McMahon, T. A. 1996 a A hydrodynamic model of locomotion in the Basilisk lizard. Nature 380, 340-342.

GlasheEn, J. W. \& MCMahon, T. A. $1996 b$ Size dependence of water-running ability in Basilisk lizards Basiliscus basiliscus. J. Exp. Biol. 199, 2611-2618.

Hinton, H. E. 1976 Plastron respiration in bugs and beetles. J. Insect Physiol. 22, 1529-1550.

Holdgate, M. W. 1955 The wetting of insect cuticle by water. J. Exp. Biol. pp. 591-617.

HsieH, T. S. 2003 Three-dimensional hindlimb kinematics of water running in the plumed basilisk lizard (Basiliscus plumifrons). J. Exp. Biol. 206, 4363-4377.

HsieH, T. S. 2004 Running on water: three-dimensional force generation by basilisk lizards. Proc. Natl Acad. Sci. 101, 16784-16788.

Hu, D. L. \& Bush, J. W. M. 2005 Meniscus-climbing insects. Nature 437, 733-736.

Hu, D. L., Chan, B. \& Bush, J. W. M. 2003 The hydrodynamics of water strider locomotion. Nature 424, 663-666.

Hu, D. L., Prakash, M., Chan, B. \& Bush, J. W. M. 2007 Water-walking devices. Exp. Fluids 43, 769-778.

JANSSENS, F. 2005 Checklist of the Collembola of the world: note on the morphology and origin of the foot of the Collembola. http://www.Collembola.org /publicat/unguis.htm.

Keller, J. B. 1998 Surface tension force on a partly submerged body. Phys. Fluids 10, 3009-3010.

Lighthill, J. 1978 Waves in Fluids. Cambridge University Press.

Linsenmair, K. E. \& JANDER, R. 1976 Das 'entspannungsschwimmen' von Velia and Stenus. Naturwissenschaften 50, 231.

Mansfield, E. H., Sepangi, H. R. \& Eastwood, E. A. 1997 Equilibrium and mutual attraction or repulsion of objects supported by surface tension. Philos. Trans. R. Soc. Lond. A 355, 869-919.

Matsuda, K., Watanabe, S. \& Eiju, T. 1985 Real-time measurement of large liquid surface deformation using a holographic shearing interferometer. Appl. Opt. 24 (24), 4443-4447.

Milewski, P. A. \& VANDEn-BroecK, J. M. 1999 Time-dependent gravity-capillary flows past an obstacle. Wave Mot. 29, 63-79.

Мгчамото, S. 1955 On a special mode of locomotion utilizing surface tension at the water-edge in some semiaquatic insects. Kontŷu 23, 45-52.

Noble-Nesbitt, J. 1963 Transpiration in Podura aquatica L. (Collembola, Isotomidae) and the wetting properties of its cuticle. J. Exp. Biol. 40, 681-700.

Plateau, J. 1873 Statique Expérimentale et Théorique des Liquides Soumis aux Seules Forces Moléculaires. Gauthier-Villars.

Prakash, M. \& Bush, J. W. M. Interfacial propulsion by directional adhesion. Nat. Materials (submitted).

Qú́ré, D. 2008 Wetting and Roughness. Annu. Rev. Mater. Res. 38, 71-99.

RAY, J. 1710 Historia insectorum. Impensis A. \& J. Churchill. 
Reyssat, M., Pépin, A., Marty, F., Chen, Y. \& Quéré, D. 2006 Bouncing transitions in microtextured materials. Europhys. Lett. 74, 306-312.

SCHILDKNECHT, H. 1976 Chemical ecology - a chapter of modern natural products chemistry. Angew. Chem. Intl Ed. Engl. 15, 214-222.

SCriven, L. E. \& Sternling, C. V. 1970 The Marangoni effects. Nature 187, 186-188.

SonG, Y. S., SuHR, S. H. \& SitTI, M. 2006 Modeling of the supporting legs for designing a biomimetic water strider robot. In Proceedings of the IEEE International Conference on Robotics and Automation, pp. 2303-2310.

Spedding, G. R., Rosén, M. \& Hedenstrom, A. 2003 A family of vortex wakes generated by a thrush nightingale in free flight in a wind tunnel over its entire natural range of flight speeds. J. Exp. Biol. 206, 2313-2344.

Spilhaus, A. 1948 Raindrop size, shape and falling speed. J. Atmos. Sci. 5 (3), 108-110.

Stratton, G. E., Suter, R. B. \& Miller, P. R. 2004a Evolution of water surface locomotion by spiders: a comparative approach. Biol. J. Linn. Soc. 81 (1), 63-78.

Stratton, G. E., Suter, R. B. \& Miller, P. R. $2004 b$ Taxonomic variation among spiders in the ability to repel water: surface adhesion and hair density. J. Arachnol. 32, 11-21.

Suhr, S. H., Song, Y. S., LeE, S. J. \& SitTi, M. 2005 Biologically inspired miniature water strider robot. Proc. Robot. Sci. Sys. pp. 42-48.

Sun, S. M. \& Keller, J. B. 2001 Capillary-gravity wave drag. Phys. Fluids 13 (8), 2146-2151.

SUTER, R. B. 2003 Trichobothrial mediation of an aquatic escape response: directional jumps by the fishing spider. J. Insect Sci. 3, 1-7.

Suter, R. B. \& Gruenwald, J. 2000 Predator avoidance on the water surface? Kinematics and efficacy of vertical jumping by Dolomedes (Araneae, Pisauridae). J. Arachnol. 28 (2), 201-210.

Suter, R. B., Rosenberg, R. B., Loeb, S., Wildman, H. \& Long, J. 1997 Locomotion on the water surface: propulsive mechanisms of the fisher spider Dolomedes triton. J. Exp. Biol. 200, $2523-2538$.

Suter, R. B., Stratton, G. \& Miller, P. 2003 Water surface locomotion by spiders: distinct gaits in diverse families. J. Arachnol. 31 (3), 428-432.

Suter, R. B. \& Wildman, H. 1999 Locomotion on the water surface: hydrodynamic constraints on rowing velocity require a gait change. J. Exp. Biol. 202, 2771-2785.

TANEDA, S. 1991 Visual observations of the flow around a half-submerged oscillating sphere. J. Fluid. Mech. 227, 193-209.

TAYlor, G. K., Nudds, R. L. \& Thomas, A. L. R. 2003 Flying and swimming animals cruise at a Strouhal number tuned for high power efficiency. Nature 425, 707-711.

ThORPE, W. H. \& CRISP, D. J. 1947 Studies on plastron respiration. Part I. The biology of Apelocheirus [Hemiptera, Aphelocheiridae (Naucoridae)] and the mechanism of plastron rentention. J. Exp. Biol. 24, 227-269.

VANDEN-BroecK, J. \& Dias, F. 1992 Gravity-capillary solitary waves in water of infinite depth and related free-surface flows. J. Fluid Mech. 240, 549-557.

Vogel, S. 1994 Life in Moving Fluids. Princeton University Press.

VoGel, S. 2006 Living in a physical world. Part VIII. Gravity and life in water. J. Biosci. 30 (3), 309-322.

Voropayev, S. I. \& Afanasyev, Y. D. 1994 Vortex Structures in a Stratified Fluid, pp. 35-37. Chapman \& Hall.

Wenzel, R. N. 1936 Resistance of solid surfaces to wetting by water. Indus. Engng Chem. 28, 988-994.

Wilga, C. \& Lauder, G. 2002 Function of the heterocercal tail in sharks: quantitative wake dynamics during steady horizontal swimming and vertical maneuvering. J. Exp. Biol. 205, $2365-2374$.

Yu, Y., Guo, M., LI, X. \& Zheng, Q. S. 2007 Meniscus-climbing behaviour and its minimum free-energy mechanism. Langmuir 23, 10546-10550. 